\title{
Review Article \\ Hamiltonian Approach to QCD in Coulomb Gauge: A Survey of Recent Results
}

\author{
H. Reinhardt $\mathbb{D}$, G. Burgio, D. Campagnari, E. Ebadati, J. Heffner, M. Quandt, \\ P. Vastag, and H. Vogt
}

Institut für Theoretische Physik, Universität Tübingen, Auf der Morgenstelle 14, 72076 Tübingen, Germany

Correspondence should be addressed to H. Reinhardt; h.reinhardt@uni-tuebingen.de

Received 6 June 2017; Revised 4 September 2017; Accepted 2 October 2017; Published 19 February 2018

Academic Editor: Ralf Hofmann

Copyright ( $) 2018$ H. Reinhardt et al. This is an open access article distributed under the Creative Commons Attribution License, which permits unrestricted use, distribution, and reproduction in any medium, provided the original work is properly cited. The publication of this article was funded by SCOAP $^{3}$

\begin{abstract}
We report on recent results obtained within the Hamiltonian approach to QCD in Coulomb gauge. Furthermore this approach is compared to recent lattice data, which were obtained by an alternative gauge-fixing method and which show an improved agreement with the continuum results. By relating the Gribov confinement scenario to the center vortex picture of confinement, it is shown that the Coulomb string tension is tied to the spatial string tension. For the quark sector, a vacuum wave functional is used which explicitly contains the coupling of the quarks to the transverse gluons and which results in variational equations which are free of ultraviolet divergences. The variational approach is extended to finite temperatures by compactifying a spatial dimension. The effective potential of the Polyakov loop is evaluated from the zero-temperature variational solution. For pure Yang-Mills theory, the deconfinement phase transition is found to be second order for $\mathrm{SU}(2)$ and first order for $\mathrm{SU}(3)$, in agreement with the lattice results. The corresponding critical temperatures are found to be $275 \mathrm{MeV}$ and $280 \mathrm{MeV}$, respectively. When quarks are included, the deconfinement transition turns into a crossover. From the dual and chiral quark condensate, one finds pseudocritical temperatures of $198 \mathrm{MeV}$ and $170 \mathrm{MeV}$, respectively, for the deconfinement and chiral transition.
\end{abstract}

\section{Introduction}

One of the most challenging problems in particle physics is the understanding of the phase diagram of strongly interacting matter. By means of ultrarelativistic heavy ion collisions the properties of hadronic matter at high temperature and/or density can be explored. From the theoretical point of view we have access to the finite-temperature behavior of QCD by means of lattice Monte-Carlo calculations. Due to the sign problem, this method fails, however, to describe baryonic matter at high density or, more technically, QCD at large chemical baryon potential [1]. Therefore, alternative, nonperturbative approaches to QCD which do not rely on the lattice formulation and hence do not suffer from the notorious sign problem are desirable. In recent years, much effort has been devoted to develop nonperturbative continuum approaches. These are based on either Dyson-Schwinger equations [2-7] or functional renormalization group flow equations $[8,9]$, or they exploit the variational principle in either the Hamiltonian $[10,11]$ or covariant $[12,13]$ formulation of gauge theory. There are also semiphenomenological approaches assuming a massive gluon propagator [14] or the Gribov-Zwanziger action [15]; see [16].

In this talk, I will review some recent results obtained within the Hamiltonian approach to QCD in Coulomb gauge both at zero and at finite temperatures; for earlier reviews, see $[17,18]$. After a short introduction to the basic features of this approach, I will summarize the essential zero-temperature results for pure Yang-Mills theory and compare them to recent lattice data which were obtained by an alternative gauge-fixing method, which is expected to yield results closer to the continuum theory. After that, I will show by means of lattice calculations that the so-called Coulomb string tension is linked not to the temporal but to the spatial string tension. In this context, I will demonstrate that the Gribov-Zwanziger confinement scenario is related to the 
center vortex picture of confinement. I will then report on new variational calculations carried out for the quark sector of QCD. After that I will extend the Hamiltonian approach to QCD in Coulomb gauge to finite temperatures by compactifying a spatial dimension [19]. Numerical results will be given for the Polyakov loop and the chiral and dual quark condensates. Finally, I will give some outlook on future research within the Hamiltonian approach.

\section{Variational Hamiltonian Approach to Yang-Mills Theory}

For pedagogical reason let me first summarize the basic features of the Hamiltonian approach in Coulomb gauge for pure Yang-Mills theory. The Hamiltonian approach to Yang-Mills theory starts from Weyl gauge $A_{0}(\mathbf{x})=0$ and considers the spatial components of the gauge field $A_{i}^{a}(\mathbf{x})$ as coordinates. The momenta are introduced in the standard fashion $\Pi_{i}^{a}(\mathbf{x})=\delta S_{\mathrm{YM}}[A] / \delta \dot{A}_{i}^{a}(\mathbf{x})=-E_{i}^{a}(\mathbf{x})$ and turn out to be the color electric field $\mathbf{E}^{a}(\mathbf{x})$. The classical Yang-Mills Hamiltonian is then obtained as

$$
H=\frac{1}{2} \int d^{3} x\left(\mathbf{E}^{2}(\mathbf{x})+\mathbf{B}^{2}(\mathbf{x})\right),
$$

where

$$
B_{k}^{a}(\mathbf{x})=\varepsilon_{k l m}\left(\partial_{l} A_{m}^{a}(\mathbf{x})-\frac{g}{2} f^{a b c} A_{l}^{b}(\mathbf{x}) A_{m}^{c}(\mathbf{x})\right)
$$

is the non-Abelian color magnetic field with $g$ being the coupling constant. The theory is quantized by replacing the classical momentum $\Pi_{i}^{a}$ with the operator $\Pi_{i}^{a}(\mathbf{x})=$ $-\mathrm{i} \delta / \delta A_{i}^{a}(\mathbf{x})$. The central issue is then to solve the Schrödinger equation $H \phi[A]=E \phi[A]$ for the vacuum wave functional $\phi[A]$. Due to the use of Weyl gauge, Gauß's law $\widehat{\mathbf{D}} \cdot \Pi \phi[A]=$ 0 (with $\widehat{\mathbf{D}}=\boldsymbol{\partial}+g \mathbf{A}$ being the covariant derivative in the adjoint representation) has to be put as a constraint on the wave functional, which ensures the gauge invariance of the latter. Instead of working with explicitly gauge invariant states, it is more convenient to fix the gauge and explicitly resolve Gauß's law in the chosen gauge. This has the advantage that any (normalizable) wave functional $\phi[A]$ is physically admissible for a variational approach, while the price to pay is a significant complication of the gauge-fixed Hamiltonian. A particular convenient choice of gauge for this method turns out to be Coulomb gauge $\boldsymbol{\partial} \cdot \mathbf{A}=0$.

After canonical quantization in Weyl gauge $A_{0}=0$ and resolution of Gauß's law in Coulomb gauge $\boldsymbol{\partial} \cdot \mathbf{A}=0$ one finds the following gauge-fixed Hamiltonian [20]:

$$
H=H_{\mathrm{T}}+H_{\mathrm{C}}
$$

with

$$
\begin{aligned}
& H_{\mathrm{T}}=\frac{1}{2} \int d^{3} x\left(J^{-1}[A] \Pi^{a}(\mathbf{x}) \cdot J[A] \Pi^{a}(\mathbf{x})+\mathbf{B}^{a}(\mathbf{x})\right. \\
& \left.\cdot \mathbf{B}^{a}(\mathbf{x})\right),
\end{aligned}
$$

where

$$
J[A]=\operatorname{Det}(-\widehat{\mathbf{D}} \cdot \boldsymbol{\partial})
$$

is the Faddeev-Popov determinant and

$$
\begin{aligned}
H_{\mathrm{C}} & =\frac{g^{2}}{2} \int d^{3} x \int d^{3} y J[A]^{-1} \rho^{a}(\mathbf{x}) J[A] \\
& \cdot\left[(-\widehat{\mathbf{D}} \cdot \boldsymbol{\partial})^{-1}\left(-\boldsymbol{\partial}^{2}\right)(-\widehat{\mathbf{D}} \cdot \boldsymbol{\partial})^{-1}\right]^{a b}(\mathbf{x}, \mathbf{y}) \rho^{b}(\mathbf{y})
\end{aligned}
$$

is the so-called Coulomb term with the color charge density

$$
\begin{aligned}
\rho^{a}(\mathbf{x}) & =f^{a b c} \mathbf{A}^{b}(\mathbf{x}) \cdot \Pi^{c}(\mathbf{x})+\rho_{m}^{a}(\mathbf{x}) \\
& \equiv \rho_{\mathrm{YM}}^{a}(\mathbf{x})+\rho_{m}^{a}(\mathbf{x}) .
\end{aligned}
$$

This expression contains besides the charge density of the matter fields $\rho_{m}^{a}$ also a purely gluonic part. Due to the implementation of Coulomb gauge, the scalar product in the Hilbert space of wave functionals $\phi[A]=\langle A \mid \phi\rangle$ is defined by

$$
\langle\phi|\cdots| \psi\rangle=\int \mathscr{D A J}[A] \phi^{*}[A] \cdots \psi[A] .
$$

Here, the functional integration is over transverse spatial gauge fields and the Faddeev-Popov determinant $J[A]$ appears due to Coulomb gauge fixing with the standard Faddeev-Popov method. The Faddeev-Popov determinant (5) in the integration measure represents the Jacobian of the change of variables from "Cartesian" to "curvilinear" variables in Coulomb gauge. With the gauge-fixed Hamiltonian (3) one has to solve the stationary Schrödinger equation $H \phi[A]=$ $E \phi[A]$ for the vacuum wave functional $\phi[A]$. Once $\phi[A]$ is known, all observables and correlation functions can, in principle, be calculated. This has been attempted by means of the variational principle using Gaussian type ansatz for the vacuum wave functional $[21,22]$. However, the first attempts did not properly include the Faddeev-Popov determinant, which turns out to be crucial in order to describe the confinement properties of the theory. Below, I will discuss the variational approach developed in [10,11], which differs from previous attempts by the ansatz for the vacuum wave functional, the treatment of the Faddeev-Popov determinant and, equally important, by the renormalization; see [23] for further details.

2.1. Variational Solution of the Schrödinger Equation. The ansatz for the vacuum wave functional is inspired by the quantum mechanics of a particle in a spherically symmetric potential for which the ground state wave function is given by $\phi(r)=u(r) / r$, where the radial wave functional $u(r)$ satisfies a standard one-dimensional Schrödinger equation and $r$ represents (the square root of the radial part of) the Jacobian of the transformation from the Cartesian to spherical coordinates. Our ansatz for the vacuum wave functional is given by

$$
\begin{aligned}
& \phi_{\mathrm{YM}}[A]=\frac{1}{\sqrt{J[A]}} \\
& \cdot \exp \left[-\frac{1}{2} \int d^{3} x \int d^{3} y A_{k}^{a}(\mathbf{x}) \omega(\mathbf{x}, \mathbf{y}) A_{k}^{a}(\mathbf{y})\right] \\
& \equiv \frac{1}{\sqrt{J[A]}} \widetilde{\phi}_{\mathrm{YM}}[A] .
\end{aligned}
$$




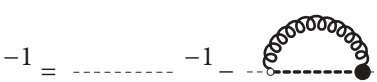

FIGURE 1: Dyson-Schwinger equation for the ghost propagator.

The inclusion of the preexponential factor has the advantage that it eliminates the Faddeev-Popov determinant from the integration measure in the scalar product (8). Furthermore, for the wave function (9), the gluon propagator is given up to a factor of $1 / 2$ by the inverse of the variational kernel $\omega(\mathbf{x}, \mathbf{y})$. As the numerical calculation shows [24] in the Yang-Mills sector the Coulomb term $H_{\mathrm{C}}(6)$ can be ignored. The reason is the presence of the Faddeev-Popov determinant in the pure Yang-Mills term of $H_{C}$ (see (6)), which ensures that in the numerator of the expectation value the gluon energy $\omega(\mathbf{p})$ occurs only in the combination $\omega(\mathbf{p})-\chi(\mathbf{p})$ (see [10]), which is infrared vanishing. The Faddeev-Popov determinant $J[A]$, however, drops out from $H_{\mathrm{C}}$ in the quark sector, since $J[A]$ commutes with the charge density $\rho_{m}^{a}(\mathbf{x})$ of the quarks; see (31).

Calculating the expectation value of the remaining parts of the Yang-Mills Hamiltonian (4) with the wave functional (9) up to two loops, the minimization of the energy density with respect to $\omega(\mathbf{x}, \mathbf{y})$ yields the following gap equation in momentum space (Due to translational and rotational invariance, kernels such as $\omega(\mathbf{x}, \mathbf{y})$ can be Fourier transformed as $\omega(\mathbf{x}, \mathbf{y})=\int\left(d^{3} k /(2 \pi)^{3}\right) \mathrm{e}^{\mathrm{i} \mathbf{k} \cdot(\mathbf{x}-\mathbf{y})} \omega(k)$ where the new kernel in momentum space depends on $k=|\mathbf{k}|$ only. For simplicity, we will use the same symbol for the kernel in position and momentum space and go back and forth between both representations with impunity.):

$$
\omega^{2}(k)=\mathbf{k}^{2}+\chi^{2}(k)+c,
$$

where $c$ is a finite renormalization constant resulting from the tadpole and

$$
\begin{aligned}
\chi_{k l}^{a b}(\mathbf{x}, \mathbf{y}) & =-\frac{1}{2}\left\langle\phi\left|\frac{\delta^{2} \ln J[A]}{\delta A_{k}^{a}(\mathbf{x}) \delta A_{l}^{b}(\mathbf{y})}\right| \phi\right\rangle \\
& =\delta^{a b} t_{k l}(\mathbf{x}-\mathbf{y}) \chi(\mathbf{x}-\mathbf{y})
\end{aligned}
$$

represents the ghost loop $\left(t_{k l}(\mathbf{x})=\delta_{k l}-\partial_{k} \partial_{l} / \partial^{2}\right.$ is the transverse projector). This can be expressed in terms of the ghost propagator:

$$
G(\mathbf{x}, \mathbf{y})=\left\langle\phi\left|(-\widehat{\mathbf{D}} \cdot \boldsymbol{\partial})^{-1}(\mathbf{x}, \mathbf{y})\right| \phi\right\rangle,
$$

which is evaluated with the vacuum wave functional (9) in the rainbow-ladder approximation, resulting in a Dyson-Schwinger equation for the form factor

$$
d(\mathbf{k})=g \mathbf{k}^{2} G(\mathbf{k})
$$

of the ghost propagator which is diagrammatically illustrated in Figure 1. This equation has to be solved together with the gap equation (10).

Dyson-Schwinger equations are functional differential equations and their solutions are uniquely determined only after providing appropriate boundary conditions. In the present case, the so-called horizon condition

$$
d^{-1}(0)=0
$$

is assumed, which is the key point in Gribov's confinement scenario. Its physical implications will be discussed later. The equations given in (10) and Figure 1 can be studied analytically in the infrared using power law ansatz:

$$
\begin{aligned}
& \omega(p)=A p^{-\alpha}, \\
& d(p)=B p^{-\beta} .
\end{aligned}
$$

Assuming a bare ghost-gluon vertex and the horizon condition (14), one finds, for the IR exponents of gluon and ghost form factor (15), the sum rule

$$
\alpha=2 \beta-(d-2),
$$

where $d$ is the number of spatial dimensions (i.e., $d=3$ is our real world). The coupled gluon gap equation (10) and ghost DSE (Figure 1) allow for a single solution in $d=2$,

$$
\beta=0.4,
$$

and for two solutions in $d=3$,

$$
\begin{aligned}
& \beta=1, \\
& \beta=0.796 .
\end{aligned}
$$

The numerical solutions of the gluon gap and ghost DSE are shown in Figure 2. The numerical solutions reproduce the result (18) of the IR analysis. At large momenta, the gluon energy $\omega(p)$ approaches the photon energy $|\mathbf{p}|$ in agreement with asymptotic freedom, while $\omega(p)$ diverges like $\sim 1 /|\mathbf{p}|$ in the IR, which implies the absence of free gluons in the IR and signals confinement.

Alternative to the variational approach, one can indirectly determine the vacuum wave functional by solving the functional renormalization group flow equations for the various propagators and vertex functions of the Hamiltonian approach. Restricting the flow equations to those for the ghost and gluon propagators, one finds for the ghost form factor the result shown in Figure 3. Starting with a constant ghost form factor in the ultraviolet, the ghost form factor develops an infrared singularity as the momentum cutoff of the flow equation tends to zero. This is nicely seen in Figure 3(b), which shows a cut through Figure 3(a) at fixed renormalization group scale $k$.

Let us also mention that it is not necessary to assume the horizon condition (14) in the case of $D=2+1$ dimensions, where it is a direct consequence of the coupled equations for the ghost and gluon propagators obtained from the variational principle. Finally, the horizon condition (14) is also seen in the lattice data for the ghost form factor; see Section 3.

2.2. Physical Implications of the Ghost Form Factor. As can be seen from its definition (13), the ghost form factor expresses the deviation of Yang-Mills theory from QED, where the 


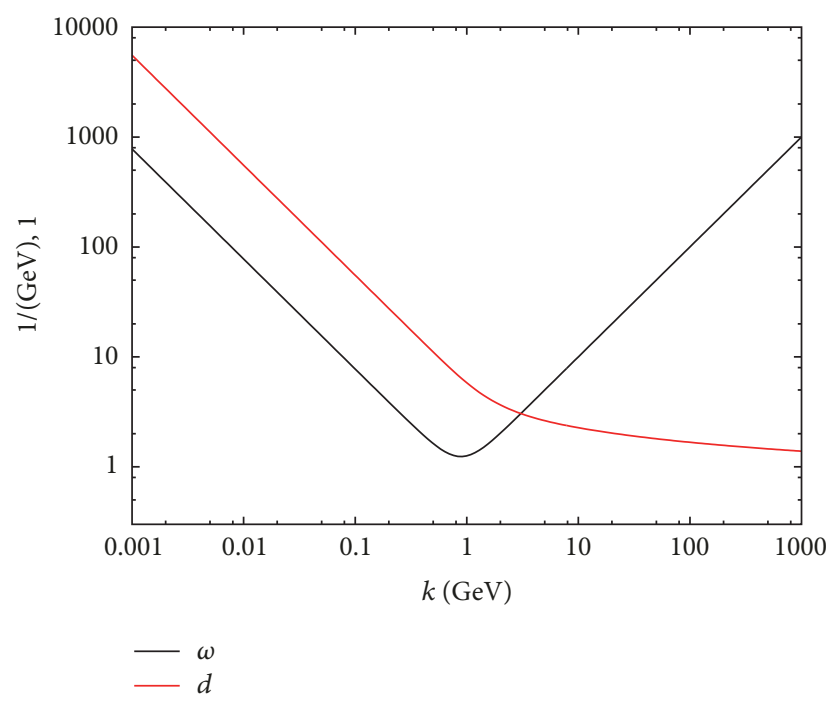

FIGURE 2: Numerical solution of the coupled gap equation for $\omega$ (10) and Dyson-Schwinger equation for the ghost form factor $d$ (13) for the renormalization constant $c=0$ for $d=3$ spatial dimensions [25]. The scale is fixed by the Coulomb string tension; see Section 4.

Faddeev-Popov operator in Coulomb gauge is given by the Laplacian, that is, the ghost propagator is $G(p)=1 / p^{2}$.

Coulomb gauge is called a physical gauge since in QED the remaining transverse components are the gauge invariant degrees of freedom. This is not the case for Yang-Mills theory. However, Coulomb gauge can be viewed as a physical gauge also in the case of Yang-Mills theory in the sense that the inverse ghost form factor in Coulomb gauge represents the dielectric function of the Yang-Mills vacuum [32]:

$$
\epsilon(k)=d^{-1}(k) \text {. }
$$

The horizon condition (14) guarantees that this function vanishes in the infrared, $\epsilon(k=0)=0$. This implies that the Yang-Mills vacuum is a perfect color dielectric, that is, a dual superconductor. In this way, the Hamiltonian approach in Coulomb gauge relates Gribov's confinement scenario to the dual Meißner effect, a confinement mechanism realized through the condensation of magnetic monopoles and proposed by Mandelstam and 't Hooft $[33,34]$. The dielectric function obtained here as inverse ghost form factor is also in accord with the phenomenological bag model picture of hadrons. Inside the hadron, that is, at small distance, the dielectric function is that of a normal vacuum while outside the physical hadrons the vanishing of the dielectric constant implies the absence of free color charges by Gauß's law.

\section{Comparison with Lattice Calculation}

Let us now compare the results of the variational solution with lattice calculations. Figure 4 shows the gluon energy and the ghost form factor in $d=2$ spatial dimension obtained in the variational approach [27] together with the lattice data [28]. The agreement is in general quite satisfactory, in particular, in the IR and the UV. There are, however, significant deviations in the mid-momentum regime. A similar picture is obtained in $d=3$ [35]. Figure 5 shows the static gluon propagator $D=1 /(2 \omega)$ in Coulomb gauge obtained in $S U(2)$ gauge theory in $d=3$. It is remarkable that the lattice data can be nicely fitted by Gribov's formula [15] (see Figure 7):

$$
\omega(p)=\sqrt{p^{2}+\frac{M^{4}}{p^{2}}}
$$

where $M$ is the so-called Gribov mass. Using a Wilsonian string tension of $\sigma_{W}=(440 \mathrm{MeV})^{2}$ one finds $M \simeq 880 \mathrm{MeV}$. The variational calculations reproduce the infrared behavior of the lattice propagator perfectly and are also in reasonably agreement with the lattice data in the ultraviolet. However, in the mid-momentum regime, some strength is missing in the variational calculation. This missing strength is the result of the Gaussian type ansatz for the vacuum wave functional. In [36], the ansatz for the vacuum wave functional was extended to include also cubic and quartic terms of the gauge field in the exponent of the vacuum wave functional:

$$
\begin{aligned}
\phi[A] \sim & \exp [-S[A]], \\
S[A]= & \frac{1}{2} \int A \omega A+\frac{1}{3 !} \int \gamma^{(3)} A A A \\
& +\frac{1}{4 !} \int \gamma^{(4)} A A A A,
\end{aligned}
$$

and one finds the full curve in Figure 5, which gives a much better agreement with the lattice data in the mid-momentum regime. Let us stress that the inclusion of the cubic and quartic terms in the exponent of the vacuum wave functional does not change the IR behavior of the gluon propagator, which is determined exclusively by the ghost loop $\chi(\mathbf{p})$.

The lattice calculation of the ghost form factor $d(p)$ (13) is more involved than that of the gluon propagator since it requires the inversion of the Faddeev-Popov operator $(-\widehat{\mathbf{D}}$. $\partial$ ), which requires high numerical accuracy for field configurations near the Gribov horizon, where the Faddeev-Popov operator has a very small eigenvalue. It turns out that the lattice results for the ghost form factor depend on how the Coulomb gauge is implemented on the lattice. In principle, this is done by maximizing the gauge-fixing functional

$$
F_{t}[g]=\sum_{\mathbf{x}, i} \operatorname{Re} \operatorname{tr} U_{i}^{g}(t, \mathbf{x}) \longrightarrow \max
$$

with respect to all spatial gauge transformations $g(\mathbf{x})$. In (22) the summation is over all spatial links at a fixed time $t$ and the maximization is performed at all lattice times. In the continuum limit the extremum condition $\delta F[g] / \delta g(\mathbf{x})=0$ yields the Coulomb gauge $\boldsymbol{\partial} \cdot \mathbf{A}=0$.

The lattice gauge-fixing condition yields a gauge copy which lies within the first Gribov region, but usually not within the fundamental modular region composed of those copies which give the absolute maximum of the functional (22). Therefore, in practice, one repeatedly performs random gauge transformations and selects in the end, that is, after Coulomb gauge fixing, the gauge copy which yields the largest maximum of the gauge-fixing functional (22). This copy is 

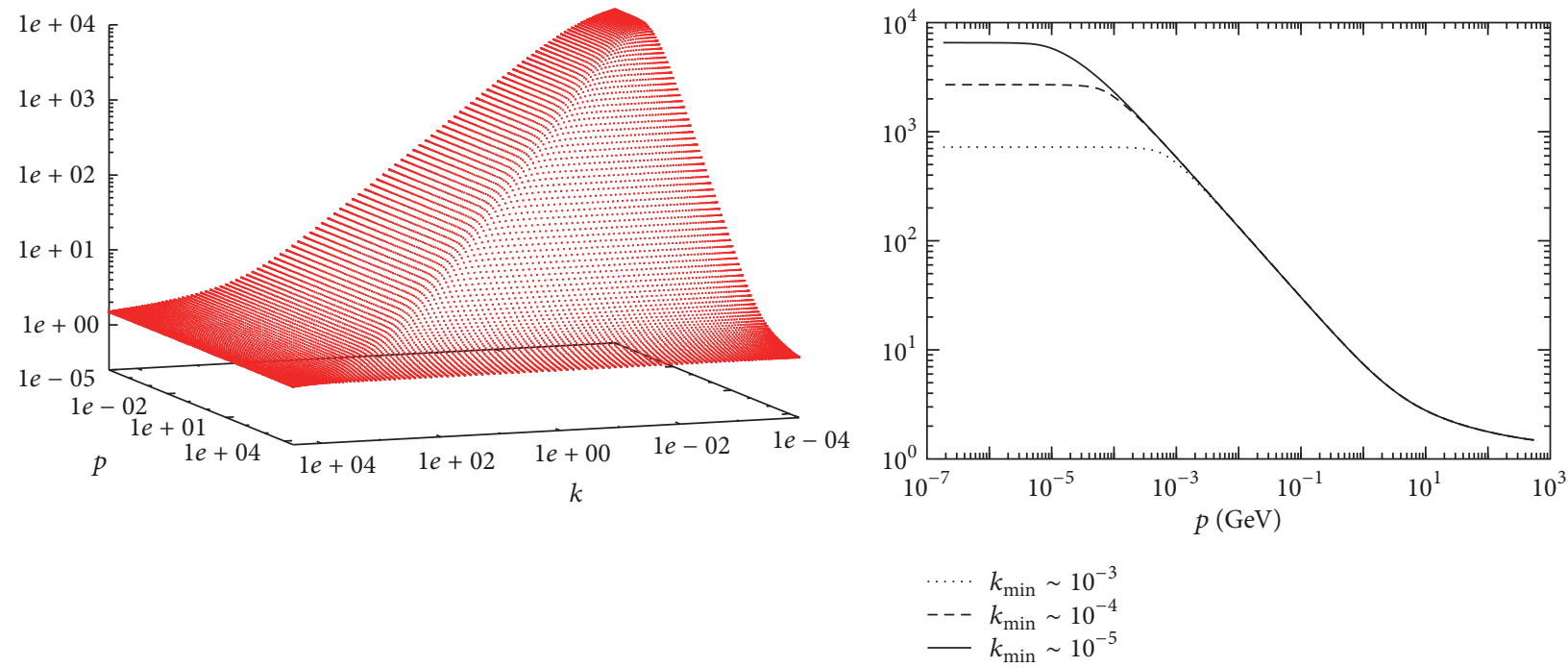

(a)

(b)

FIgURE 3: (a) The ghost form factor obtained in [26] from the solution of the renormalization group flow equations. Here, $p$ represents the momentum variable of the ghost form factor while $k$ is the infrared momentum cutoff of the flow equations. (b) Cuts through subfigure (a) at various values of the momentum scale $k$ of the flow equations.

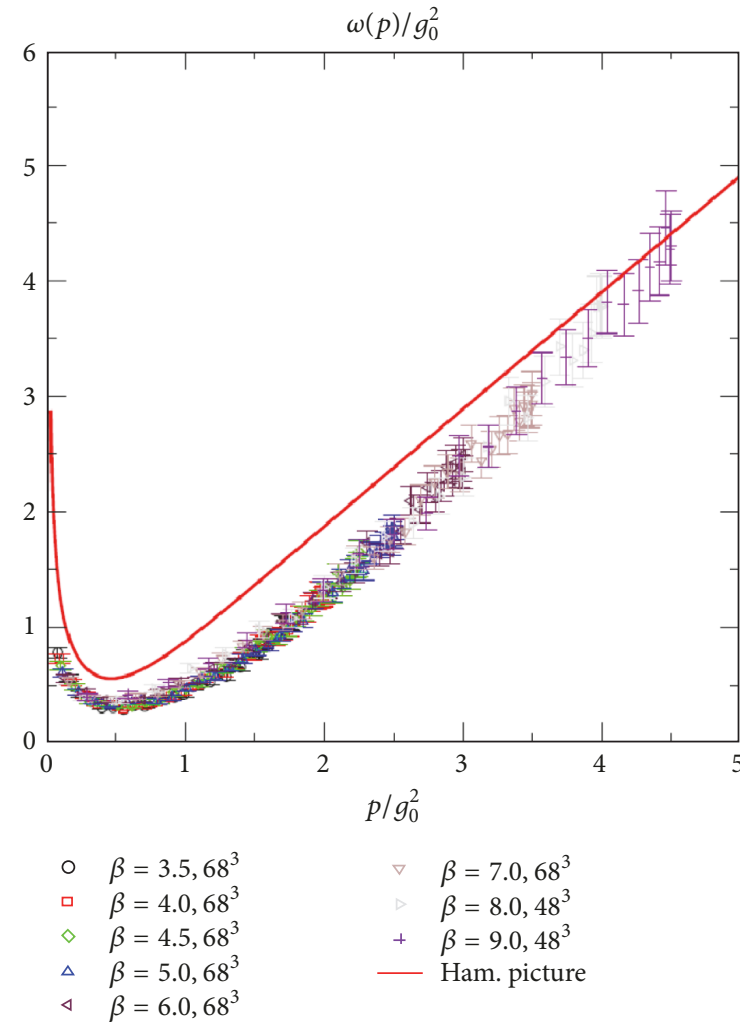

(a)

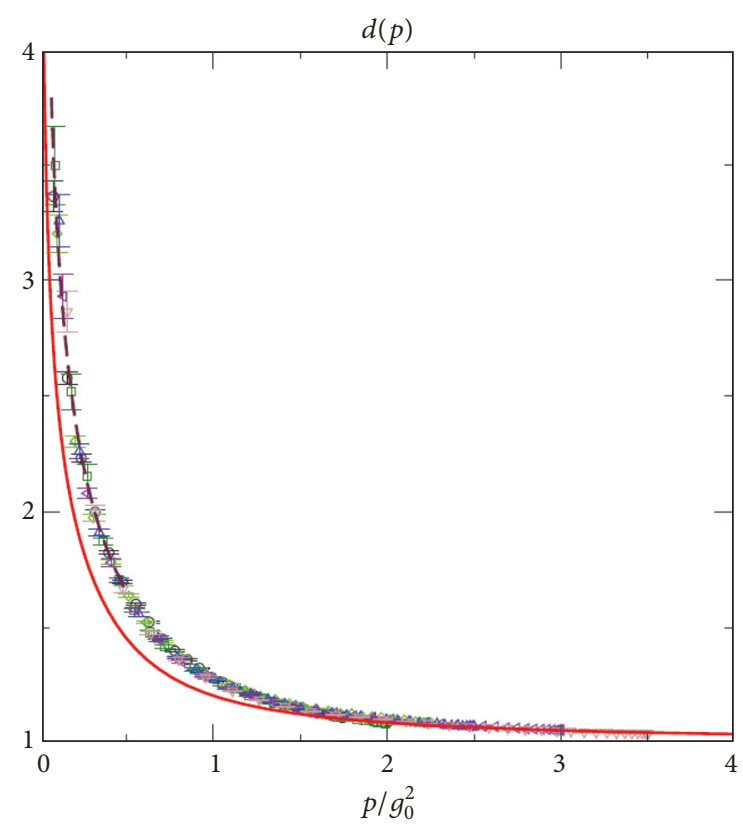
○ $\beta=3.5$
$\square \beta=4.0$
$\diamond \beta=4.5$
$\triangle \beta=5.0$
$\triangleleft \beta=6.0$
$\nabla \beta=7.0$
- Ham. picture
- - - IR fit

(b)

Figure 4: Comparison of the variational approach to $(2+1)$-dimensional Yang-Mills theory in Coulomb gauge [27] with the lattice data [28]: (a) gluon energy and (b) ghost form factor. 


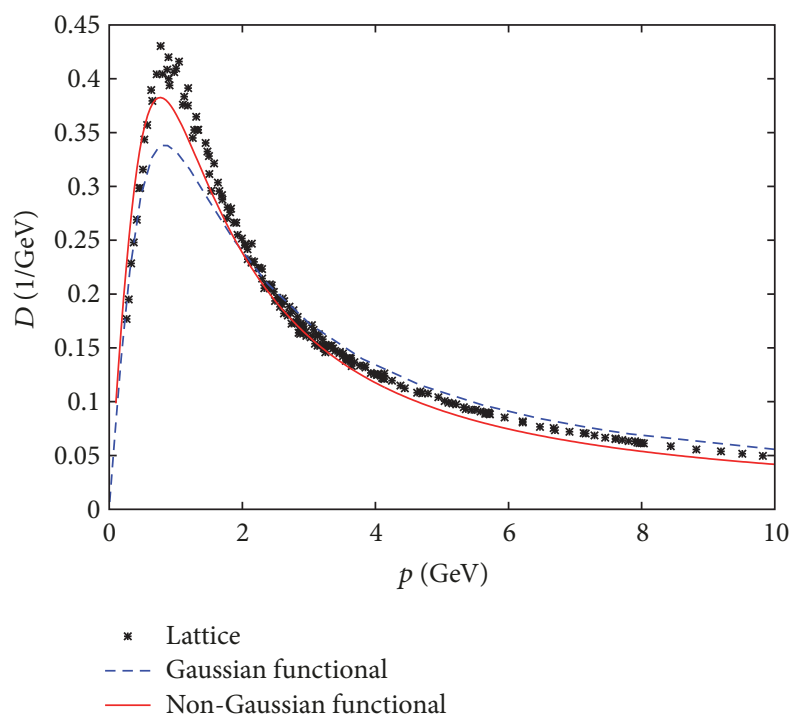

FIgURE 5: The static gluon propagator in Coulomb gauge calculated on the lattice for $\mathrm{SU}(2)$ gauge theory (crosses). The dashed and the full curves show the result of the variational calculation using, respectively, a Gaussian and non-Gaussian ansatz for the vacuum wave functional.

called "best copy" since it is assumed that this method yields a gauge copy which is the best representative of the global maximum. Figure 6 shows the result for the ghost form factor using the "best copy" gauge fixing. The obtained ghost form factor has an IR exponent of $\beta \simeq 0.5$, which is at odds with the sum rule (16) given that an IR exponent of $\alpha=1$ is obtained for the lattice gluon propagator; see (20). This result is puzzling since the sum rule is considered incontrovertible as it is obtained under quite mild assumptions. However, in [37], it was shown that for the $U(1)$ lattice gauge theory on $S^{2}$ the "best copy" method does not necessarily provide the best approximation to the fundamental modular region. An alternative lattice gauge-fixing method consists in choosing not the "best" Gribov copy but that gauge copy which minimizes the lowest eigenvalue of the Faddeev-Popov operator [38]. This configuration is referred to as the "lowest" Gribov copy. As argued in [39], the "lowest copy" method should yield results closer to the continuum theory. We have used the "lowest copy" (lc) method to recalculate the ghost and gluon propagator; see [40]. While the gluon propagator is basically the same as obtained with the "best copy" (bc) method (see Figure 7), the ghost form factor gets further enhanced in the IR as the number of gauge-fixing attempts increases (see Figure 8). (To find the absolute extremum in the bc and lc approach, we have repeated the gauge-fixing procedure a large number $N_{r}=10, \ldots, 10000$ of times, starting each time from a different random gauge transformation of the original configuration. In general, the number $N_{r}$ of gaugefixing trials is indicative of the number of Gribov copies included, even though the exact relation is complicated and nonlinear [40].) Although we did not find a strict saturation for a sufficiently large number of gauge-fixing attempts, the IR exponent of the ghost form factor is compatible with the continuum result of $\beta \simeq 1$ (see Figure 9), in agreement with the sum rule (16).

\section{The Coulomb String Tension}

The Coulomb term $H_{\mathrm{C}}$ (6) plays an important role in the Gribov-Zwanziger confinement scenario. Its Yang-Mills vacuum expectation value

$$
V_{\mathrm{C}}=g^{2}\left\langle(-\widehat{\mathbf{D}} \cdot \boldsymbol{\partial})^{-1}\left(-\boldsymbol{\partial}^{2}\right)(-\widehat{\mathbf{D}} \cdot \boldsymbol{\partial})^{-1}\right\rangle
$$

provides an upper bound for the potential between static point-like color charges and is referred to as (non-Abelian) Coulomb potential. The Coulomb potential found within the variational approach $[10,11,25]$ is shown in Figure 10(a). At small distances it behaves like an ordinary Coulomb potential, $V_{\mathrm{C}}(r) \sim 1 / r$, and increases linearly at large distances with a coefficient given by the so-called Coulomb string tension $\sigma_{\mathrm{C}}$. It was shown in [41] that this quantity is an upper bound to the Wilsonian string tension $\sigma_{\mathrm{W}}$. On the lattice one finds $\sigma_{\mathrm{C}} / \sigma_{\mathrm{W}} \approx 2 \cdots 4[29,42,43]$. Due to the constraint $\sigma_{\mathrm{C}} \geq \sigma_{\mathrm{W}}$ in the Gribov-Zwanziger confinement scenario a necessary condition for confinement is that the non-Abelian Coulomb potential (23) rises at least linearly at large distances.

One may now ask, what field configurations induce the horizon condition, $d^{-1}(0)=0$, and the linearly rising Coulomb potential $V_{\mathrm{C}}(23)$ and thus confinement? Given the relation of Gribov's confinement scenario to the dual superconductor, we expect magnetic monopoles to play a substantial role. Lattice calculations carried out in the socalled indirect maximum center gauge, which contains the maximum Abelian gauge in an intermediate step, showing that magnetic monopoles are tied to center vortices [44]. This can be also understood in the continuum [45]. Center vortices are string-like gauge field configurations in $D=3$ or world surfaces in $D=4$, for which the Wilson loop equals a nontrivial center element of the gauge group, provided the loop has nontrivial linking with the center vortices. (By the Bianchi identity center vortices form closed loops in $D=3$ and closed surfaces in $D=4$.)

Lattice calculations provide strong evidence that confinement is due to center vortices. Indeed, when the center vortex content of the gauge field configurations is removed, one finds that the Wilsonian string tension and thus confinement disappears [46].

On the lattice center vortices can be detected as follows [47]: one first brings the gauge field configurations into the so-called maximal center gauge:

$$
\sum_{x, \mu}\left|\operatorname{tr} U_{\mu}^{2}(x)\right| \longrightarrow \max
$$

which rotates a link as close as possible to a center element, for example, $Z_{\mu}(x)= \pm 1 \in Z(2)$ for the gauge group $\mathrm{SU}(2)$. Subsequently, one performs a so-called center projection:

$$
U_{\mu}(x) \longrightarrow Z_{\mu}(x)
$$

which replaces each link with its nearest center element. One is then left with $Z(2)$ links, which form closed center 


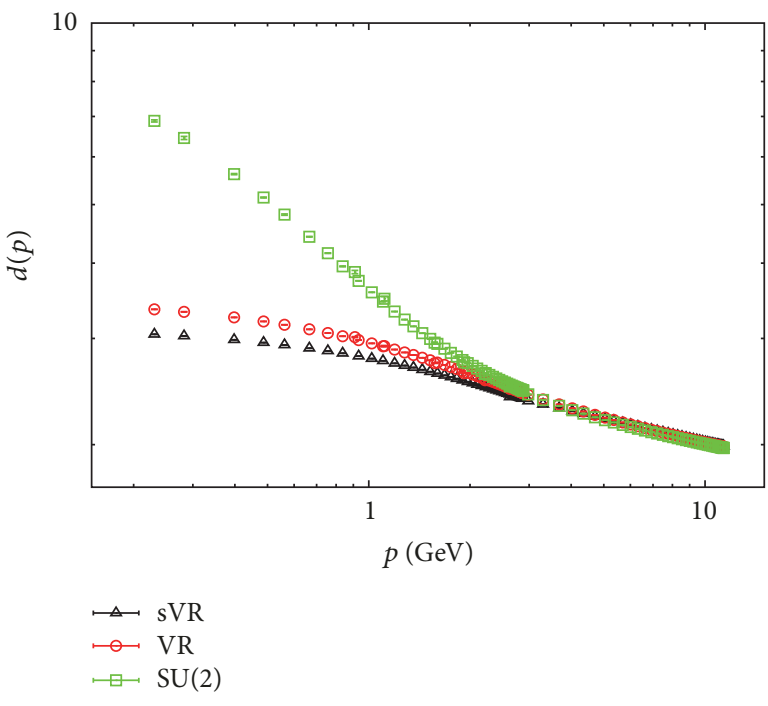

(a)

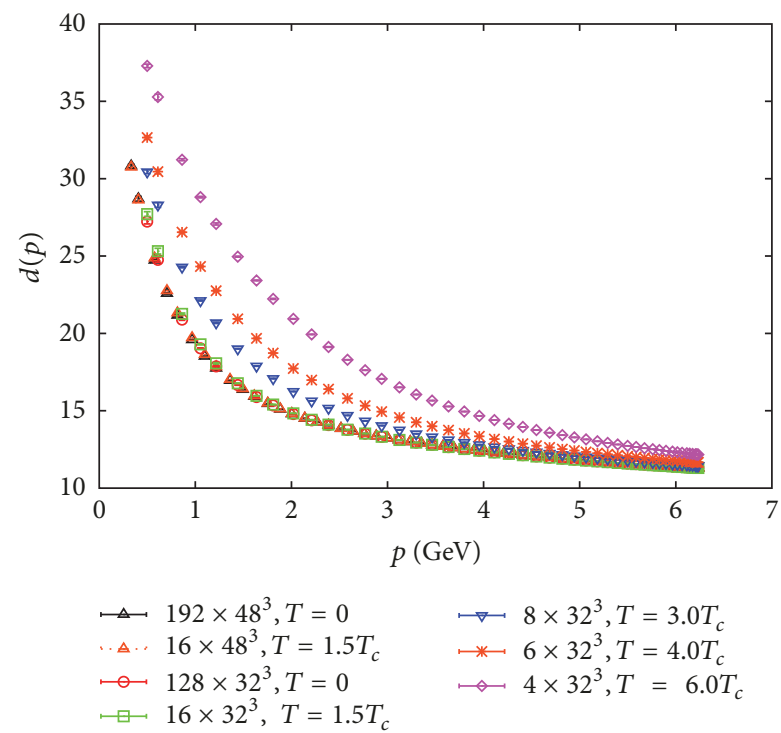

(b)

Figure 6: (a) The ghost form factor in Coulomb gauge calculated on the lattice in [29] (green squares). The red circles and black triangles show the results obtained for the ghost form factor when all center vortices or only the spatial center vortices are removed from the ensemble of gauge field configurations; see main text. (b) Ghost form factor calculated on the lattice for different temperatures.

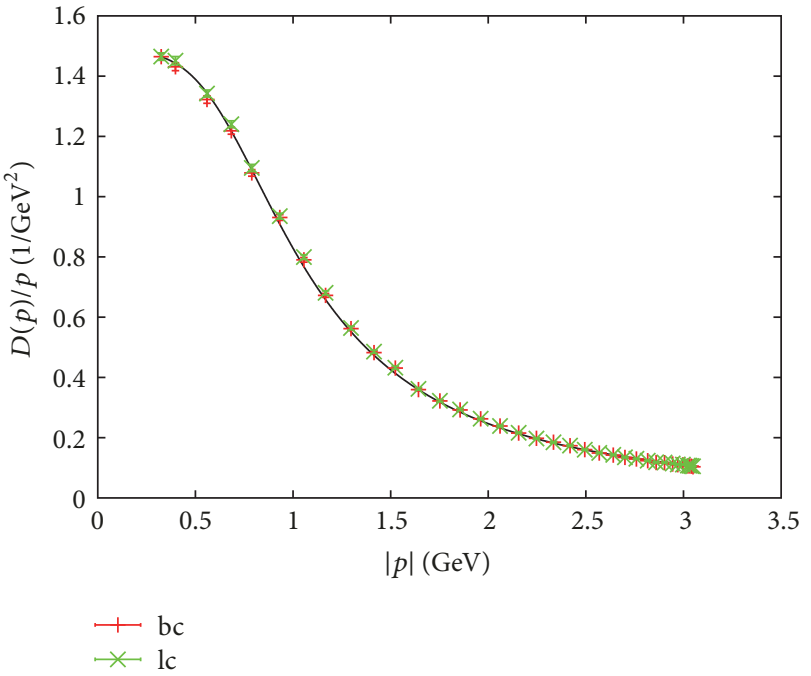

Figure 7: The gluon propagator with the bc and the lc approach from 1000 gauge-fixing attempts. The solid line is a fit to the Gribov formula (20). The choice of Gribov copies apparently makes no visible difference.

vortices, the only nontrivial field configurations in a $Z(2)$ theory. When a center vortex pierces a Wilson loop it contributes a nontrivial center element to the latter. It was shown in [48] that the center vortices obtained in this way are physical objects in the sense that they show the proper scaling behavior; that is, their area density survives the continuum limit. This property distinguishes the center vortices found after center projection in the maximal center gauge from other gauges like, for example, the Laplacian center gauge [44].
The center vortex content of a gauge field configuration can be removed [46] by multiplying the original link variable $U_{\mu}(x)$ by its center projection $Z_{\mu}(x)$ :

$$
U_{\mu}(x) \longrightarrow U_{\mu}(x) \cdot Z_{\mu}(x) .
$$

Figure 6 shows the ghost form factor obtained on the lattice when the center vortices are removed from the ensemble of gauge field configurations as described above [29]. The ghost form factor becomes infrared flat and the horizon condition is lost. This shows that center vortices induce the horizon condition which is the cornerstone of Gribov's confinement scenario. This also shows that Gribov's confinement scenario is tied to the center vortex picture of confinement. This is in accord with the observation that center vortices and magnetic monopoles are located on the Gribov horizon of Coulomb gauge [42].

When center vortices are removed as described above, the static color potential extracted from a Wilson loop loses its linearly rising part; that is, the Wilsonian string tension $\sigma_{\mathrm{W}}$ disappears after center vortex removal. Since $\sigma_{\mathrm{C}} \geq \sigma_{\mathrm{W}}$ this does not necessarily imply that elimination of center vortices also removes the Coulomb string tension. In [29], the non-Abelian Coulomb potential was calculated after center projection and center vortex removal. Removing the center vortices also eliminates the Coulomb string tension while center vortex projection keeps only the linearly rising part of the non-Abelian Coulomb potential; see Figure 10(b). This result is perhaps not so surprising since center vortices live on the Gribov horizon (more precisely on the common boundary between the Gribov horizon and the fundamental modular region [42]), which represents the domain of the infrared dominant field configurations in the Gribov-Zwanziger confinement scenario. 


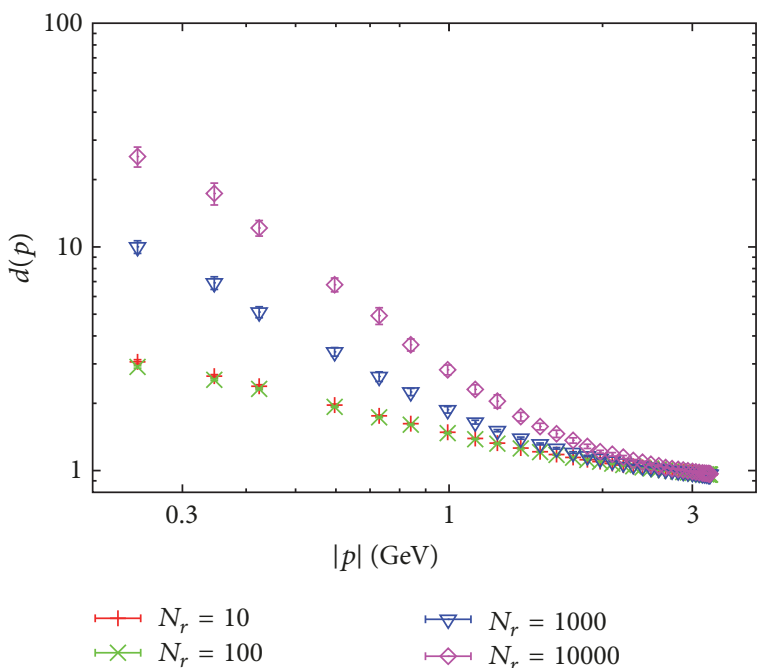

(a)

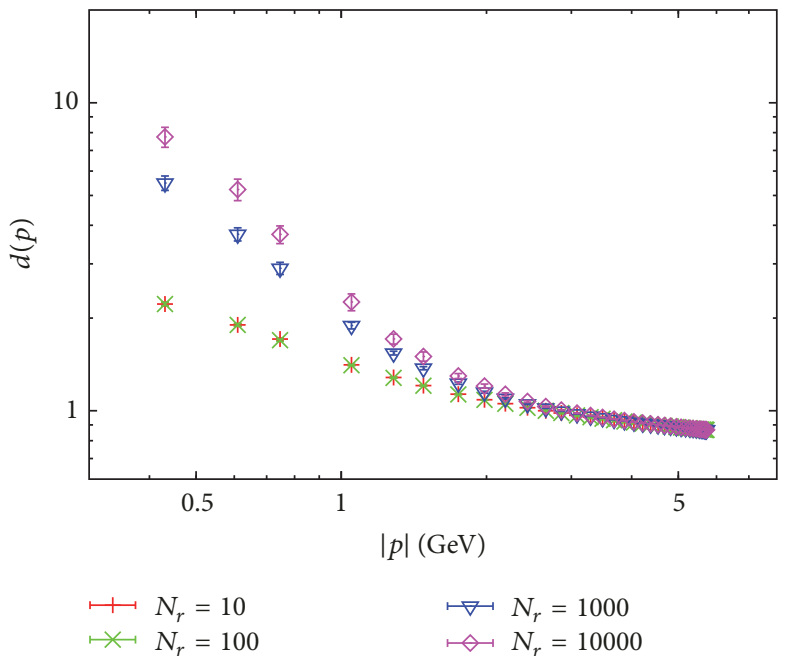

(b)

FIGURE 8: The ghost form factor after gauge fixing to the lowest-eigenvalue copy with increasing number of trials from 10 to 10000 on $24^{4}$ lattices at (a) $\beta=2.2$ and (b) $\beta=2.4$, where $\beta$ is the (inverse) lattice coupling constant.

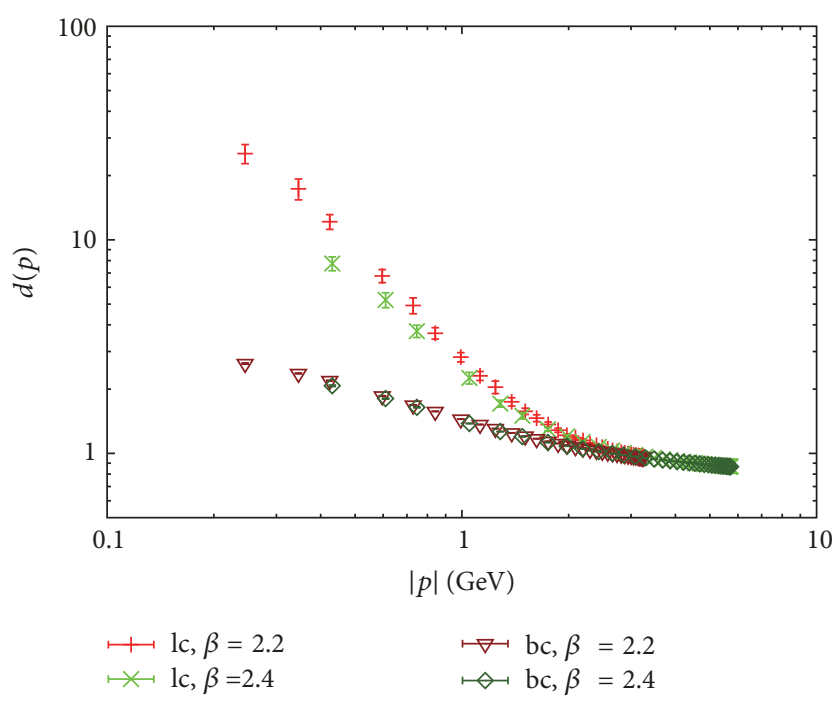

FIGURE 9: The ghost form factor after 10000 copies of bc and lc strategy.

At finite temperature different Wilsonian string tensions are measured from temporal and spatial Wilson loops referred to as temporal and spatial string tension, respectively. Above the deconfinement phase transition these two Wilsonian string tensions decouple. While the spatial string tension increases above the critical temperature, the temporal string tension disappears. On the lattice it is not difficult to see that in the center projected $Z(2)$ theory the temporal and spatial Wilsonian string tensions, that is, the area law in the temporal and spatial Wilson loop, are produced by temporal and spatial center vortices, respectively. The latter are formed exclusively by spatial center-valued links:

$$
U_{i}(x) \longrightarrow Z_{i}(x)
$$

which will be referred to as spatial center projection in the following. (In $D=3$, spatial center vortices are closed lines formed by a "stack" of spatial plaquettes with nontrivial value after center projection. Geometrically, they extend in the time direction (on the dual lattice) and can thus link with spatial Wilson loops. The terminology in $D=4$ is similar; that is, spatial center vortices are hypersurfaces on the dual lattice which are composed of spatial plaquettes on the original lattice, which are nontrivial after center projection. Geometrically, such spatial vortices extend in one space and one time direction and may hence link with spatial Wilson loops.) Analogously multiplying the spatial link by its nearest center projected $Z(2)$ element,

$$
U_{i}(x) \longrightarrow U_{i}(x) \cdot Z_{i}(x),
$$

removes all spatial center vortices and thus the spatial string tension while the temporal links are unaffected. Therefore, the temporal string tension, which can be calculated from the correlator of Polyakov loops and hence from temporal links exclusively, will not be affected by the spatial center vortex removal. Figure $11(\mathrm{a})$ shows the quantity $p^{4} V_{\mathrm{C}}(p)$ whose infrared limit gives the Coulomb string tension, $\lim _{p \rightarrow 0} p^{4} V_{C}(p)=8 \pi \sigma_{C}$. As one observes, the Coulomb string tension disappears already when only the spatial center vortices are removed. This clearly shows that the Coulomb string tension is related to the spatial string tension and not to the temporal one. This explains also the finitetemperature behavior of the Coulomb string tension, which increases with the temperature above the deconfinement phase transition just like the spatial string tension; see Figure 11(b).

A necessary condition for the Gribov-Zwanziger confinement scenario to be realized is that the ghost form factor is infrared divergent, which is indeed found in the variational approach and also on the lattice; see Figure 6(b). However, 

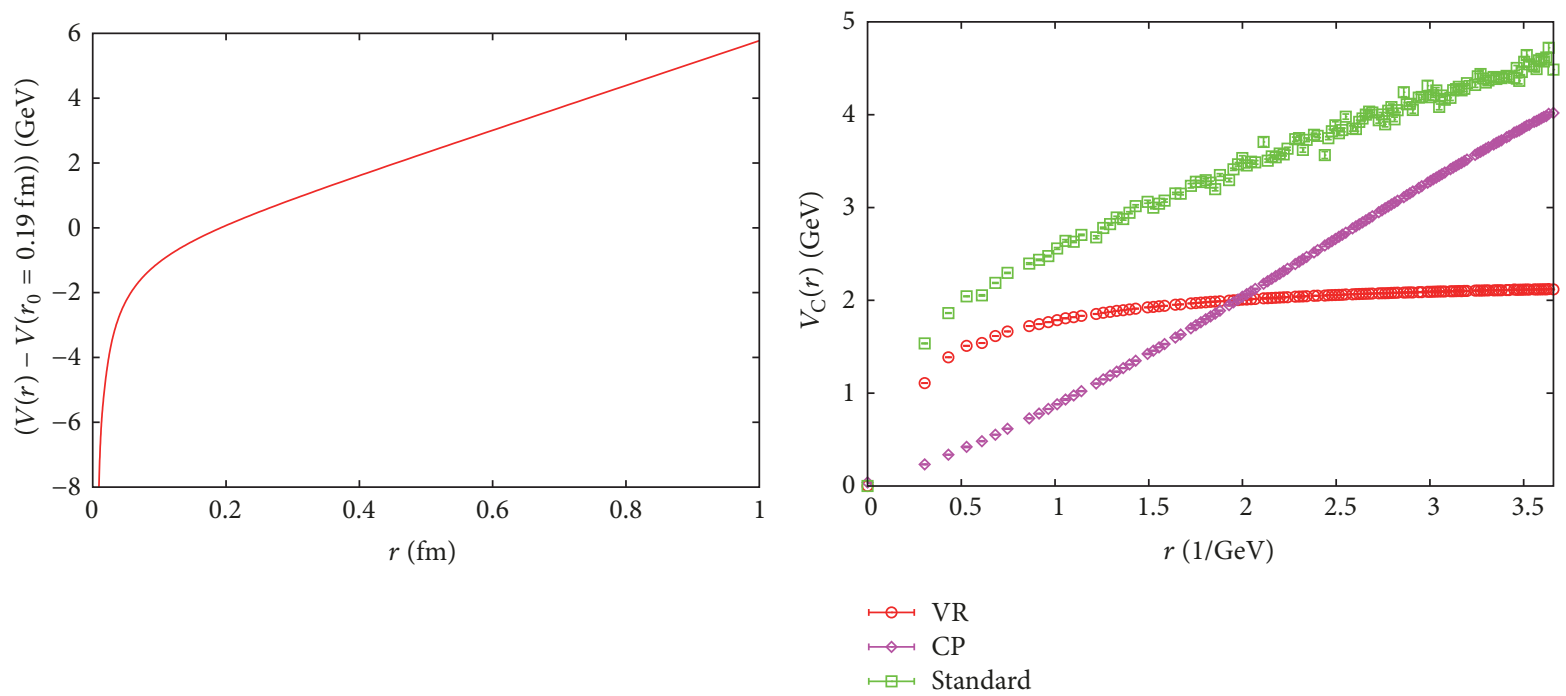

(a)

(b)

Figure 10: (a) Non-Abelian Coulomb potential (23) obtained within the variational approach [25]. (b) Standard non-Abelian Coulomb potential (green boxes) compared to the potential obtained after vortex removal (red circles) and center projection (violet diamonds) [29].

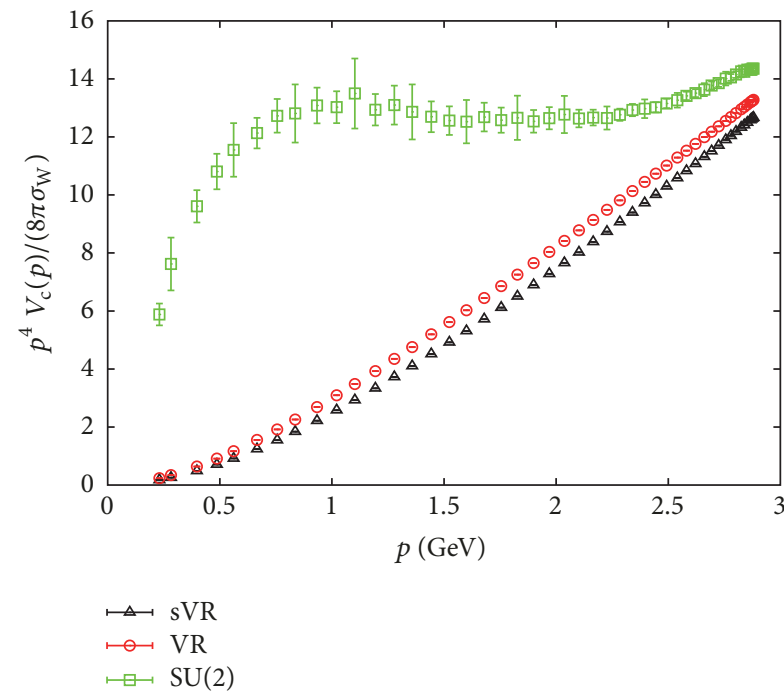

(a)

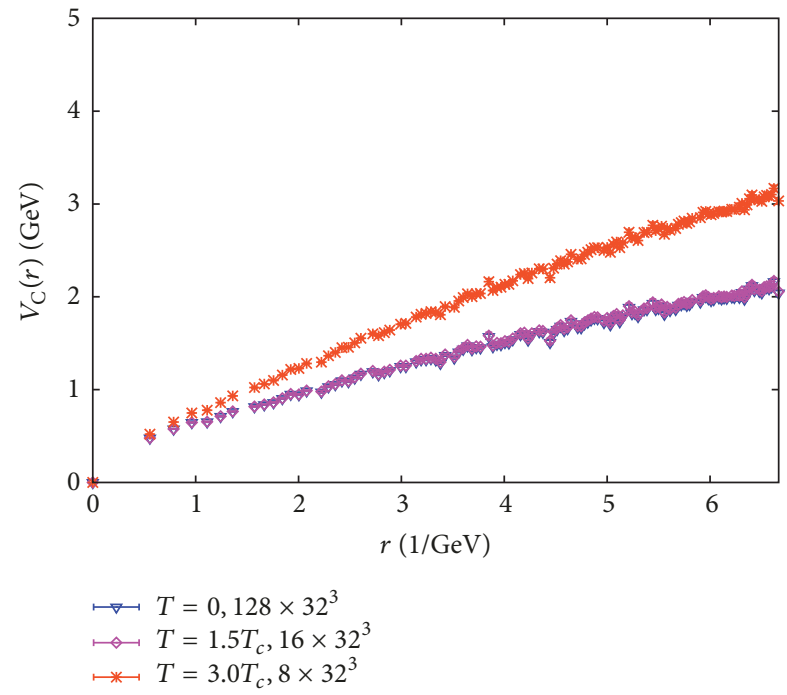

(b)

Figure 11: (a) Non-Abelian Coulomb potential in momentum space (green boxes) compared to the result obtained after removing just the spatial (black triangles) or all center vortices (red circles). (b) Non-Abelian Coulomb potential for different temperatures $\left(T_{c}\right.$ denotes the critical temperature).

the infrared divergence disappears when one removes the center vortices or the spatial center vortices only as can be seen in Figure 6(a). Also, the spatial center vortex projection produces the same ghost form factor as full center projection. This also explains why the infrared divergence of the ghost form factor does not disappear above the deconfinement phase transition. Thus both features of the Gribov-Zwanziger confinement scenario, the infrared divergent ghost form factor and the linearly rising Coulomb potential, are caused by spatial center vortices and are thus tied to the spatial string tension, which increases above the deconfinement transition.

\section{Variational Approach to the Quark Sector}

The variational approach to Yang-Mills theory in Coulomb gauge presented in Section 2 has been extended to full QCD 
in $[30,49,50]$. The Hamiltonian of full QCD in Coulomb gauge is given by

$$
H_{\mathrm{QCD}}=H_{\mathrm{T}}+H_{\mathrm{Q}}+H_{\mathrm{C}}
$$

where $H_{\mathrm{T}}$ is the Hamiltonian of the transverse gluon degrees of freedom (4), $H_{\mathrm{C}}$ is the Coulomb interaction (6), and

$$
\begin{aligned}
& H_{\mathrm{Q}} \\
& \quad=\int d^{3} x \psi^{\dagger}(\mathbf{x})\left[\boldsymbol{\alpha} \cdot\left(-\mathrm{i} \nabla+g t^{a} \mathbf{A}^{a}(\mathbf{x})\right)+\beta m_{0}\right] \psi(\mathbf{x})
\end{aligned}
$$

is the Hamiltonian of the quarks coupling to the transverse gluon field. Here, $\boldsymbol{\alpha}, \beta$ are the usual Dirac matrices, $t^{a}$ denotes the generator of the color group in the fundamental representation, and $m_{0}$ is the bare current quark mass (of electroweak origin) which will be neglected in the following. Furthermore, when the quarks are included, the matter charge density in the Coulomb Hamiltonian $H_{C}(7)$ is given by

$$
\rho_{m}^{a}(\mathbf{x})=\psi^{\dagger}(\mathbf{x}) t^{a} \psi(\mathbf{x})
$$

In $[30,50]$, the quark sector of QCD has been treated within the variational approach using the following ansatz for the QCD wave functional

$$
|\phi[A]\rangle=\mathcal{N} \frac{1}{\sqrt{I[A]}} \phi_{\mathrm{YM}}[A]\left|\phi_{\mathrm{Q}}[A]\right\rangle
$$

where $\phi_{\mathrm{YM}}$ is the Yang-Mills vacuum functional (9) and

$$
\begin{aligned}
& \left|\phi_{\mathrm{Q}}[A]\right\rangle \\
& \quad=\exp \left[-\int d^{3} x \int d^{3} y \psi_{+}^{\dagger}(\mathbf{x}) K(\mathbf{x}, \mathbf{y}) \psi_{-}(\mathbf{y})\right]|0\rangle,
\end{aligned}
$$

with

$$
\begin{aligned}
& K(\mathbf{x}, \mathbf{y}) \\
& =\beta S(\mathbf{x}, \mathbf{y}) \\
& \quad+g \int d^{3} z[V(\mathbf{x}, \mathbf{y} ; \mathbf{z})+\beta W(\mathbf{x}, \mathbf{y} ; \mathbf{z})] \boldsymbol{\alpha} \cdot \mathbf{A}^{a}(\mathbf{z}) t^{a}
\end{aligned}
$$

being the quark wave functional. Here, $S, V$, and $W$ are variational kernels. Furthermore, $|0\rangle$ is the Fock vacuum of the quarks which represents the bare Dirac sea. Finally,

$$
I[A]=\left\langle\phi_{\mathrm{Q}}[A] \mid \phi_{\mathrm{Q}}[A]\right\rangle=\operatorname{det}\left(\mathrm{id}+K^{\dagger} K\right)
$$

is the quark determinant. The ansatz (32) treats the quark determinant $I[A]$ and the Faddeev-Popov determinant (5) on equal footing.

The ansatz (33) reduces for $W=0$ to the quark wave functional used in [49] while for $V=W=0$ it becomes the BCS-type wave functional considered in [51-53]. A comment is here in order concerning the choice of the Dirac structures in the ansatz for the vacuum wave functional (33), (34). The first coupling term $(\sim V)$ is the usual quark-gluon coupling, which also occurs in the QCD Hamiltonian (30). This coupling form is mandatory to capture the quark-gluon coupling of the QCD Hamiltonian in the vacuum expectation value. The second coupling term $(\sim W)$ was included for technical reasons. With these two coupling terms all UV divergences cancel in the variational equations; see below. There exist, of course, more Dirac structures for the quark-gluon couplings. The variational principle guarantees that the inclusion of further Dirac structures with additional variational kernels can only improve the quality of the description. With the wave functional (32) the expectation value of the QCD Hamiltonian was calculated up to two loops. Variation with respect to the two kernels $V$ and $W$, which describe the coupling of the quarks to the transverse gluons, gives two equations, which can be solved explicitly in terms of the scalar kernel $S$ and the gluon energy $\omega$ yielding

$$
\begin{aligned}
V(\mathbf{p}, \mathbf{q}) & =\frac{1+S(p) S(q)}{p P(p)\left(1-S^{2}(p)+2 S(p) S(q)\right)+q P(q)\left(1-S^{2}(q)+2 S(p) S(q)\right)+\omega(|\mathbf{p}+\mathbf{q}|)}, \\
W(\mathbf{p}, \mathbf{q}) & =\frac{S(p)+S(q)}{p P(p)\left(1-S^{2}(p)-2 S(p) S(q)\right)+q P(q)\left(1-S^{2}(q)-2 S(p) S(q)\right)+\omega(|\mathbf{p}+\mathbf{q}|)},
\end{aligned}
$$

where we have defined the quantity

$$
P(p)=\frac{1}{1+S^{2}(p)}
$$

The variational equation for the scalar kernel $S$, referred to as gap equation, is highly nonlocal and can only be solved numerically. However, one can show analytically that all UV divergences in this equation cancel: the UV-divergent contributions to $S(k)$ induced by the kernels $V$ and $W$ are given, respectively, by

$$
\begin{aligned}
& \frac{C_{\mathrm{F}}}{16 \pi^{2}} g^{2} S(k)\left[-2 \Lambda+k \ln \frac{\Lambda}{\mu}\left(-\frac{2}{3}+4 P(k)\right)\right], \\
& \frac{C_{\mathrm{F}}}{16 \pi^{2}} g^{2} S(k)\left[2 \Lambda+k \ln \frac{\Lambda}{\mu}\left(\frac{10}{3}-4 P(k)\right)\right] .
\end{aligned}
$$

Here, $C_{\mathrm{F}}=\left(N_{\mathrm{C}}^{2}-1\right) / 2 N_{\mathrm{C}}$ is the quadratic Casimir, $\Lambda$ is the UV cutoff, and $\mu$ is an arbitrary momentum scale. In the sum 
of the two terms given by (39), the linear UV divergences obviously cancel. Furthermore, the sum of the logarithmic UV divergences of these two terms cancels against the asymptotic contribution to the gap equation induced by the Coulomb kernel,

$$
-\frac{C_{\mathrm{F}}}{6 \pi^{2}} g^{2} k S(k) \ln \frac{\Lambda}{\mu} .
$$

Due to the exact cancellation of all UV divergences no renormalization of the gap equation is required. This is certainly a big advantage of the present ansatz (33) for the quark wave functional. Using the gluon propagator $\sim 1 / \omega$ obtained in the Yang-Mills sector as input, the quark gap equation can be solved within a quenched calculation. In this approach, the coupling constant $g$ is determined by fixing the chiral quark condensate to its phenomenological value [30]. Figure 12 shows the vector kernels $V(\mathbf{p}, \mathbf{q}), W(\mathbf{p}, \mathbf{q})$ obtained in this way, as function of the modulus $p=q$ of the ingoing quark momenta and the cosine of the angle between them, $z=\cos \varangle(\mathbf{p}, \mathbf{q})$. These kernels are peaked in the mid-momentum regime. Furthermore, the vector kernel $V$ is about a factor of two larger than the kernel $W$. Figure 13 shows the scalar kernel $S(p)$ and the mass function

$$
M(p)=\frac{2 p S(p)}{1-S^{2}(p)}
$$

on a logarithmic scale. For the sake of comparison we also quote the curves obtained when the coupling to the transverse gluons is neglected. More precisely, this corresponds to putting $g=0$ in the ansatz (34) and discarding the second (perturbative) part in the approximation

$$
V_{\mathrm{C}}(p) \approx \frac{8 \pi \sigma_{\mathrm{C}}}{p^{4}}+\frac{g^{2}}{p^{2}}
$$

for the Coulomb potential (see (23)). As one observes, the inclusion of the coupling to the transverse gluon changes only the mid- and large-momentum regime while the infrared behavior is not changed at all. This is perhaps a little bit surprising but should have been expected in view of the fact that the non-Abelian Coulomb term [the first part in (42)], which gives rise to a linearly rising potential at large distances and dominates the infrared behavior of the gap equation. Let us also mention that we do not find chiral symmetry breaking from our equations when the linearly rising part of the Coulomb potential is neglected.

\section{Hamiltonian Approach to Finite-Temperature QCD by Compactifying a Spatial Dimension}

In $[24,54]$ the variational approach to Yang-Mills theory in Coulomb gauge was extended to finite temperatures by making a quasiparticle ansatz for the density matrix of the grand canonical ensemble where the quasiparticle energy was determined by minimizing the free energy. The resulting variational equations could be solved analogously to the ones at zero-temperature. There is, however, a more efficient way to treat Yang-Mills theory at finite temperature within the Hamiltonian approach. The motivation comes from the Polyakov loop

$$
P\left[A_{0}\right](\mathbf{x})=\frac{1}{d_{r}} \operatorname{tr} \mathscr{P} \exp \left[\mathrm{i} g \int_{0}^{L} d x^{0} A_{0}\left(x^{0}, \mathbf{x}\right)\right],
$$

where $A_{0}=A_{0}^{a} t^{a}$ is the temporal gauge field in the fundamental representation, $\mathscr{P}$ is the path ordering prescription, $d_{r}$ denotes the dimension of the representation of the gauge group, and $L=1 / T$ is the length of the compactified Euclidean time axis which represents the inverse temperature. The Polyakov loop cannot be calculated straightforwardly in the Hamiltonian approach due to the unrestricted time interval and the use of the Weyl gauge $A_{0}=0$. Both problems are overcome in the more efficient Hamiltonian approach to finite-temperature quantum field theory developed in [19]. This approach does not require an ansatz for the density matrix of the grand canonical ensemble and allows the evaluation of the Polyakov loop. In this novel approach, one exploits the $O(4)$ invariance to interchange the Euclidean time axis with one spatial axis. The temporal (anti)periodic boundary conditions to the fields become then spatial boundary conditions, while the new (Euclidean) time axis has infinite extent as is required within the Hamiltonian approach (see below). The upshot is that the partition function at finite temperature $L^{-1}$ is entirely given by the ground state calculated on the spatial manifold $\mathbb{R}^{2} \times S^{1}(L)$, where $S^{1}(L)$ is a circle with length $L$. The whole thermodynamics of the theory is then encoded in the vacuum calculated on the partially compactified spatial manifold $\mathbb{R}^{2} \times S^{1}(L)$. This approach was used in [55] to study Yang-Mills theory at finite temperature and in [31] to calculate the Polyakov loop within the Hamiltonian approach. Furthermore, in [56], the so-called dual quark condensate was evaluated using this approach. Let us briefly sketch its main properties.

Consider finite-temperature quantum field theory in the standard functional integral approach. Here the finite temperature is introduced by going to Euclidean space and compactifying the Euclidean time dimension by imposing periodic and antiperiodic boundary conditions for Bose and Fermi fields, respectively,

$$
\begin{aligned}
& A\left(x^{0}=\frac{L}{2}\right)=A\left(x^{0}=-\frac{L}{2}\right), \\
& \psi\left(x^{0}=\frac{L}{2}\right)=-\psi\left(x^{0}=-\frac{L}{2}\right) .
\end{aligned}
$$

The length of the compactified dimension $L$ represents then the inverse temperature $T^{-1}=L$. One can now exploit the $O(4)$ invariance of the Euclidean Lagrangian to rotate the Euclidean time axis into a space axis and, correspondingly, one spatial axis into the Euclidean time axis. Of course, 


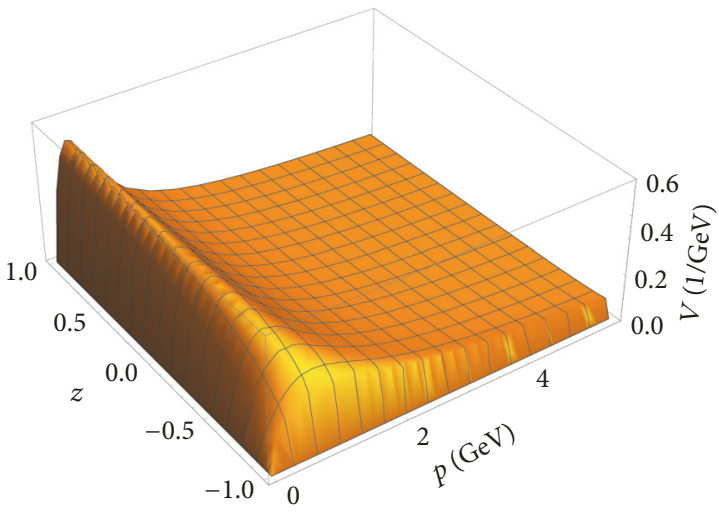

(a)

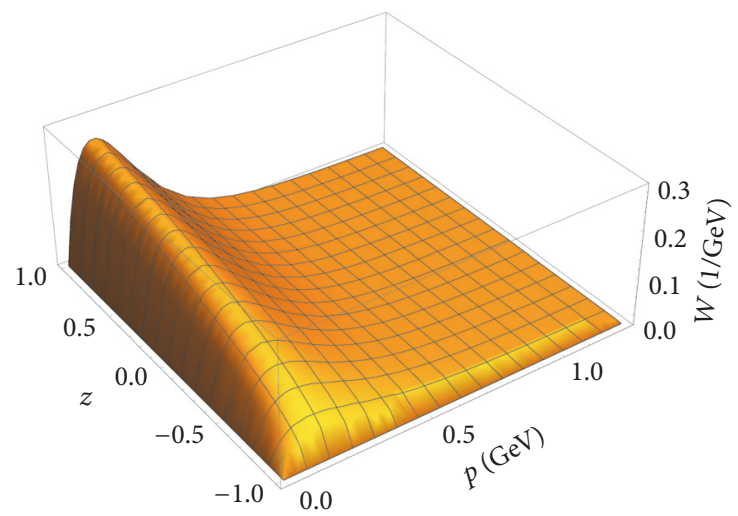

(b)

Figure 12: The vector kernel (a) $V(\mathbf{p}, \mathbf{q})(36)$ and (b) $W(\mathbf{p}, \mathbf{q})(37)$ obtained from the solution of the quark gap equation for $g \simeq 2.1$ as function of $p=q$ and $z=\cos \varangle(\mathbf{p}, \mathbf{q})$ [30].

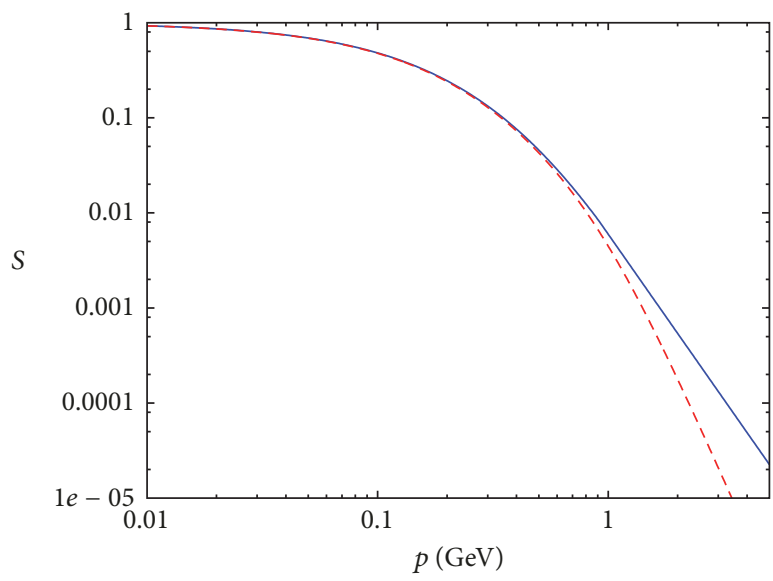

— Coupling included - - - Without coupling

(a)

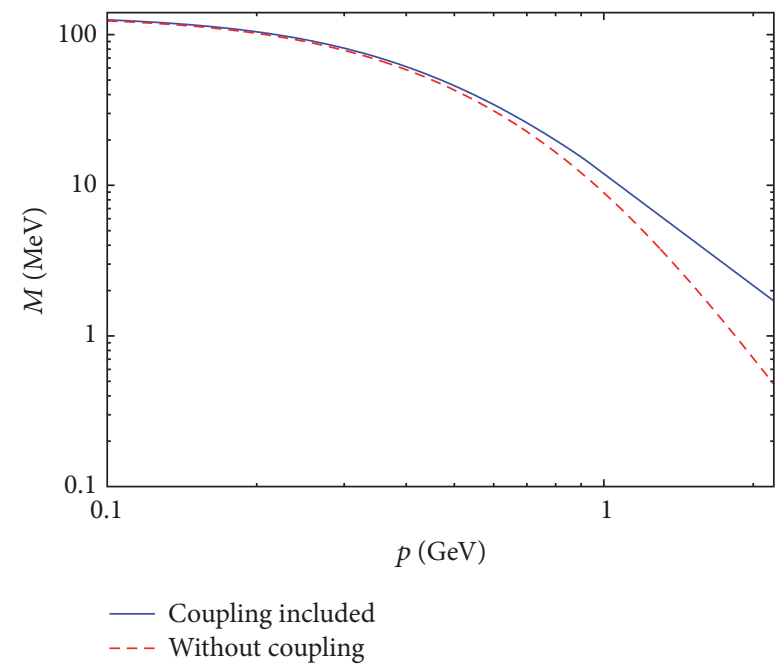

(b)

FIGURE 13: (a) Scalar form factor and (b) mass function obtained from the (quenched) solution of the quark gap equation. Results are presented for $g \simeq 2.1$ (full curve) and $g=0$ (dashed curve).

thereby all vectorial quantities transform in the same way; that is, we can choose the transformation:

$$
\begin{aligned}
& x^{0} \longrightarrow x^{3}, \\
& A^{0} \longrightarrow A^{3}, \\
& \gamma^{0} \longrightarrow \gamma^{3} \\
& x^{1} \longrightarrow x^{0}, \\
& A^{1} \longrightarrow A^{0}, \\
& \gamma^{1} \longrightarrow \gamma^{0} \\
& x^{2} \longrightarrow x^{1}, \\
& A^{2} \longrightarrow A^{1},
\end{aligned}
$$

$$
\begin{aligned}
& \gamma^{2} \longrightarrow \gamma^{1}, \\
& x^{3} \longrightarrow x^{2}, \\
& A^{3} \longrightarrow A^{2}, \\
& \gamma^{3} \longrightarrow \gamma^{2} .
\end{aligned}
$$

After this rotation we are left with the spatial periodic and antiperiodic boundary conditions:

$$
\begin{aligned}
& A\left(x^{3}=\frac{L}{2}\right)=A\left(x^{3}=-\frac{L}{2}\right), \\
& \psi\left(x^{3}=\frac{L}{2}\right)=-\psi\left(x^{3}=-\frac{L}{2}\right) .
\end{aligned}
$$


As a consequence of the $O(4)$ rotation our spatial manifold is now $\mathbb{R}^{2} \times S^{1}(L)$ instead of $\mathbb{R}^{3}$ while the temporal manifold is $\mathbb{R}$ independent of the temperature; that is, the temperature is now encoded in one spatial dimension while time has infinite extension. We can now apply the usual canonical Hamiltonian approach to this rotated space-time manifold. As the new time axis has infinite extension $\ell \rightarrow \infty$, the partition function is now given by

$$
Z(L)=\lim _{\ell \rightarrow \infty} \operatorname{tr} \exp (-\ell H(L)),
$$

where $H(L)$ is the usual Hamiltonian obtained after canonical quantization, however, now defined on the spatial manifold $\mathbb{R}^{2} \times S^{1}(L)$. Taking the trace in the basis of the exact eigenstates of the Hamiltonian $H(L)$, we obtain for the partition function (47):

$$
\begin{aligned}
Z(L) & =\lim _{\ell \rightarrow \infty} \sum_{n} \exp \left(-\ell E_{n}(L)\right) \\
& =\lim _{\ell \rightarrow \infty} \exp \left(-\ell E_{0}(L)\right) .
\end{aligned}
$$

The full partition function is now obtained from the ground state energy calculated on the spatial manifold $\mathbb{R}^{2} \times S^{1}(L)$. Introducing the energy density $e(L)$ on $\mathbb{R}^{2} \times S^{1}(L)$ by separating the volume $L \ell^{2}$ of the spatial manifold from the energy, we have

$$
E_{0}(L)=L \ell^{2} e(L)
$$

For the physical pressure

$$
P=\frac{1}{L} \frac{\partial \ln Z}{\partial V}, \quad V=\ell^{3}
$$

one finds from (48)

$$
P=-e(L)
$$

while the physical energy density $\varepsilon$ is obtained as

$$
\varepsilon=\frac{\partial(L e(L))}{\partial L}-\mu \frac{\partial e(L)}{\partial \mu} .
$$

To distinguish this quantity from the (negative) Casimir pressure $e(L)$ [see (51)], which also appears as an energy density in our formalism after the transformation [seee (45)], we will denote $e(L)$ as pseudo energy density. Finally, after the $O(4)$ rotation (45), the finite chemical potential $\mu$ enters the single-particle Dirac Hamiltonian $h$ in the following form:

$$
h(\mu)=h(\mu=0)+\mathrm{i} \mu \alpha^{3}
$$

where $\alpha^{3}$ is the third Dirac matrix and $h(\mu=0)$ the standard Dirac operator coupled to the gauge field.

6.1. Free Bose and Fermi Gases. To illustrate the above approach let us first consider a relativistic Bose gas with dispersion relation $\omega(p)=\sqrt{\mathbf{p}^{2}+m^{2}}$, where we assume for simplicity a vanishing chemical potential. The thermodynamical pressure obtained from the grand canonical ensemble for such a system is given by

$$
\begin{aligned}
& P=\frac{2}{3} \int \frac{d^{3} p}{(2 \pi)^{3}} \frac{p^{2}}{\omega(p)} n(p) \\
& n(p)=\frac{1}{\exp (\beta \omega(p))-1},
\end{aligned}
$$

where $n(p)$ are the finite temperature Bose occupation numbers. On the other hand, one finds for the ideal Bose gas with dispersion relation $\omega(p)=\sqrt{\mathbf{p}^{2}+m^{2}}$ the pseudo energy density on the spatial manifold $\mathbb{R}^{2} \times S^{1}(L)[19]$ :

$$
\begin{aligned}
e(L)=\frac{1}{2} \int \frac{d^{2} p_{\perp}}{(2 \pi)^{2}} \frac{1}{L} \sum_{n=-\infty}^{\infty} \sqrt{\mathbf{p}_{\perp}^{2}+p_{n}{ }^{2}+m^{2}}, & \\
& p_{n}=\frac{2 n \pi}{L},
\end{aligned}
$$

where $p_{n}$ are the bosonic Matsubara frequencies. This quantity does not look at all like the negative of the pressure (54), as it should by (51). In fact, as it stands it is ill-defined: the integral and the sum are both divergent. To make it mathematically well defined, we first use the proper-time regularization of the square root:

$$
\begin{aligned}
& \sqrt{A}=\frac{1}{\Gamma(-1 / 2)} \\
& \cdot \lim _{\Lambda \rightarrow \infty}\left[\int_{1 / \Lambda^{2}}^{\infty} d \tau \tau^{-3 / 2} \exp (-\tau A)-2 \Lambda+\mathcal{O}\left(\Lambda^{-1}\right)\right] .
\end{aligned}
$$

The divergent constant appears because the limit $\Lambda \rightarrow \infty$ of the incomplete $\Gamma$-function is not smooth; it drops out when taking the difference to the zero-temperature case after (59) below. With this replacement, the momentum integral in (55) can be carried out in closed form. For the remaining Matsubara sum, we use the Poisson resummation formula:

$$
\frac{1}{2 \pi} \sum_{k=-\infty}^{\infty} \exp (\mathrm{i} k x)=\sum_{n=-\infty}^{\infty} \delta(x-2 \pi n),
$$

by whose means we obtain

$$
\frac{1}{2 \pi} \int_{-\infty}^{\infty} d z f(x) \sum_{k=-\infty}^{\infty} \mathrm{e}^{\mathrm{i} k \beta z}=\frac{1}{\beta} \sum_{n=-\infty}^{\infty} f\left(\omega_{n}\right)
$$

for any function $f(z)$. The term with $k=0$ on the r.h.s. represents the zero-temperature limit of the l.h.s. After use of (58) the proper-time integral can also be carried out, yielding for the pseudo energy density (55):

$$
e(L)=-\frac{1}{2 \pi^{2}} \sum_{k=-\infty}^{\infty}\left(\frac{m}{k L}\right)^{2} K_{2}(k L m),
$$

where $K_{v}(z)$ is the modified Bessel function. The term with $k=0$ is divergent and represents the pseudo energy density of the zero-temperature vacuum, which should be eliminated 
from the pressure. The remaining terms $k \neq 0$ are all finite and also the remaining sum converges. This sum, however, cannot be carried out analytically for massive bosons (the same applies to the integral in the grand canonical expression (54) for the pressure). In the zero-mass limit, we find from (59) for the pressure (51)

$$
P=\frac{\zeta(4)}{\pi^{2}} T^{4}=\frac{\pi^{2}}{90} T^{4}
$$

which is Stefan-Boltzmann law, the correct result also obtained from the grand canonical ensemble. For massive bosons the evaluation of the sum in (59) as well as the evaluation of the integral in (54) has to be done numerically. The result is shown in Figure 14(a). As expected the pressure calculated from the compactified spatial dimension reproduces the result of the usual grand canonical ensemble. Figure 14(b) shows the various contributions to the pressure. It is seen that only a few terms in the sum of (59) are necessary to reproduce the result of the grand canonical ensemble to good accuracy.

In the case of the relativistic Fermi gas with dispersion relation $\omega(p)=\sqrt{\mathbf{p}^{2}+m^{2}}$ the energy density on $\mathbb{R}^{2} \times S^{1}(L)$ is given by

$$
\begin{array}{r}
e(L)=-2 \int \frac{d^{2} p_{\perp}}{(2 \pi)^{2}} \frac{1}{L} \sum_{n=-\infty}^{\infty} \sqrt{\mathbf{p}_{\perp}^{2}+\left(p_{n}+\mathrm{i} \mu\right)^{2}+m^{2}}, \\
p_{n}=\frac{2 n+1}{L} \pi,
\end{array}
$$

where we have now included a nonvanishing chemical potential $\mu$. To make this expression mathematically well defined one has to resort again to the proper-time regularization and Poisson resummation technique sketched above. The result is

$$
e(L)=\frac{2}{\pi^{2}} \sum_{n=0}^{\infty} \cos \left[n L\left(\frac{\pi}{L}-\mathrm{i} \mu\right)\right]\left(\frac{m}{n L}\right)^{2} K_{-2}(n L m) .
$$

Again, the term with $n=0$ represents the zero-temperature vacuum energy density, which is divergent and has to be removed. As before, this expression can only be calculated in closed form for massless particles. For the remaining sum to converge, an analytic continuation $\mathrm{i} \mu L \rightarrow \bar{\mu} \in \mathbb{R}$ is required to carry out the sum

$$
\sum_{n=1}^{\infty}(-1)^{n} \frac{\cos (n \bar{\mu})}{n^{4}}=\frac{1}{48}\left[-\frac{7}{15} \pi^{2}+2 \pi^{2} \bar{\mu}^{2}-\bar{\mu}^{4}\right] .
$$

Continuing back to real chemical potentials one finds through (51) for the pressure

$$
P=\frac{1}{12 \pi^{2}}\left[\frac{7}{15} \pi^{4} T^{4}+2 \pi^{2} T^{2} \mu^{2}+\mu^{4}\right]
$$

which is the correct result obtained also from the usual grand canonical ensemble.

In [55], the above approach was used to study Yang-Mills theory at finite temperature. For this purpose, it is merely required to repeat the variational Hamiltonian approach on the spatial manifold $\mathbb{R}^{2} \times S^{1}(L)$. Due to the one compactified spatial dimension the three-dimensional integral equations of the zero-temperature case are replaced with a set of twodimensional integral equations distinguished by different Matsubara frequencies. Below, I will use this approach to calculate the effective potential of the Polyakov loop, the order parameter of confinement.

6.2. The Polyakov Loop. Consider $\mathrm{SU}(N)$ gauge theory at finite temperature, where the temperature is introduced by the usual periodic boundary condition in the temporal directions (44a) and (44b). Gauge transformations preserving this boundary conditions need to be periodic only up to an element $z$ of the center $Z(N)$ of the gauge group,

$$
U\left(x^{0}=L\right)=z U\left(x^{0}=0\right), \quad z \in Z(N) .
$$

Since there are $N$ center elements, this theory has a residual global $Z(N)$ symmetry, which remains after gauge fixing. However, there are quantities which are sensitive to such a $Z(N)$ symmetry transformation. The most prominent example is the Polyakov loop (43). A gauge transformation of the form (65) multiplies the Polyakov loop by the center element $z$, that is,

$$
P\left[A_{0}^{U}\right]=z P\left[A_{0}\right]
$$

The expectation value of the Polyakov loop

$$
\left\langle P\left[A_{0}\right](\mathbf{x})\right\rangle \sim \exp \left(-F_{\infty}(\mathbf{x}) L\right)
$$

can be shown to be related to the free energy $F_{\infty}(\mathbf{x})$ of a static color point charge located at $\mathbf{x}$ [57]. In a confining theory this quantity has to be infinite since there are no free color charges, while in a deconfined phase it is finite. Accordingly we find for the expectation value of the Polyakov loop

$$
\left\langle P\left[A_{0}\right](\mathbf{x})\right\rangle \begin{cases}=0 & \text { confined phase } \\ \neq 0 & \text { deconfined phase. }\end{cases}
$$

From (66), it follows that the Polyakov loop vanishes in a center symmetric state. Hence, in the deconfined phase the $Z(N)$ center symmetry must be obviously broken. In the continuum theory the Polyakov loop can be most easily calculated in the Polyakov gauge:

$$
\partial_{0} A_{0}=0, \quad A_{0} \text { color diagonal. }
$$

In this gauge, one finds, for example, for the $\mathrm{SU}(2)$ gauge group, that the Polyakov loop

$$
P\left[A_{0}\right](\mathbf{x})=\cos \left(\frac{1}{2} g A_{0}(\mathbf{x}) L\right)
$$

is a one-to-one function of the gauge field, at least in the fundamental modular region of this gauge. It can be shown, see $[14,58-60]$, that instead of the expectation value of the Polyakov loop $\left\langle P\left[A_{0}\right]\right\rangle$ one may alternatively use the Polyakov loop of the expectation value, $P\left[\left\langle A_{0}\right\rangle\right]$, or the expectation value of the temporal gauge field itself, $\left\langle A_{0}\right\rangle$, as order 


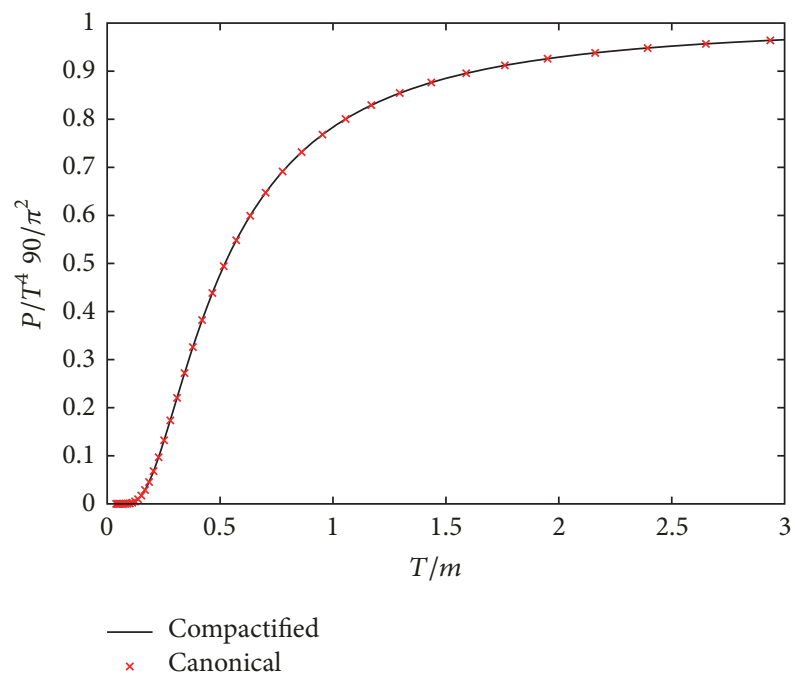

(a)

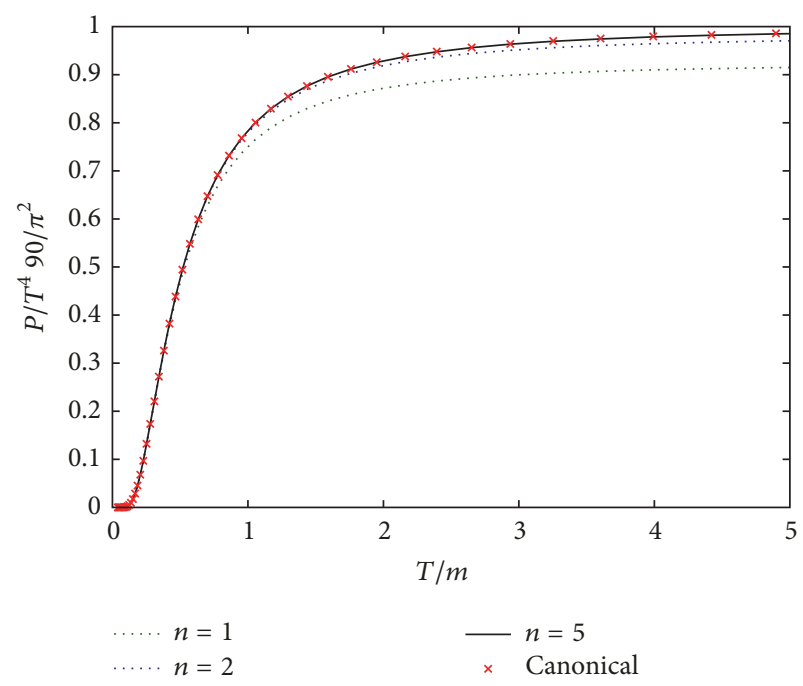

(b)

FIGURE 14: The pressure of a free massive Bose gas (a) calculated from equation (59) (full curve) and from the grand canonical ensemble (54) (crosses). (b) The pressure when the summation index in equation (59) is restricted to $|n|=1,2$ and 5.

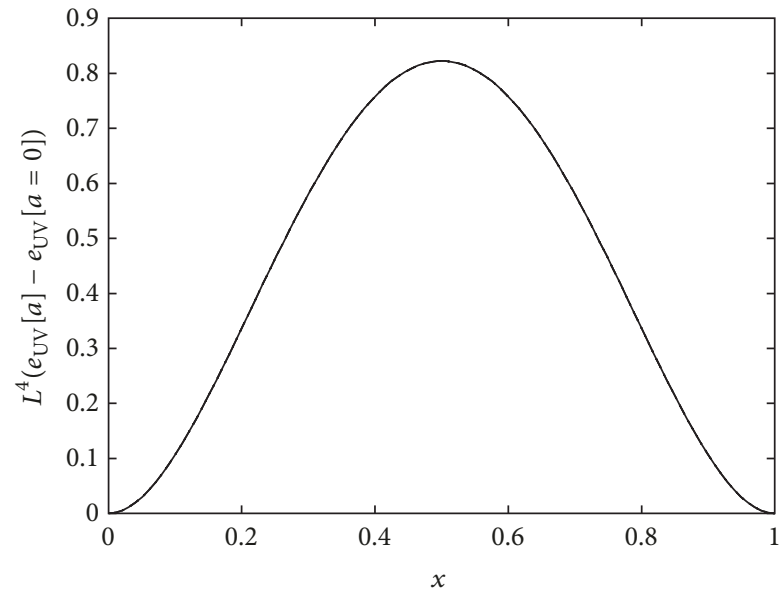

(a)

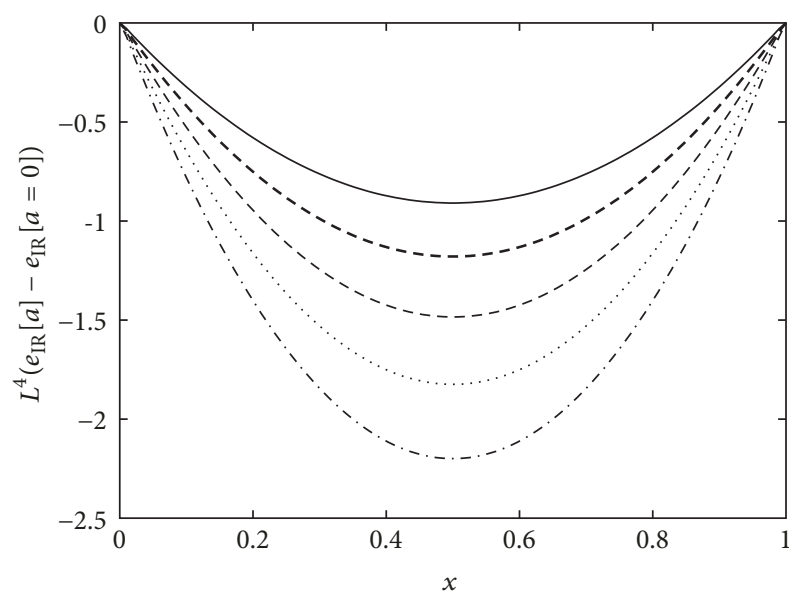

(b)

Figure 15: The effective potential of the Polyakov loop $e(a, L)(71)$ as function of the background field $x=a_{3} L / 2 \pi$. The curvature is neglected $(\chi=0)$ and the gluon energy assumed to be (a) $\omega(p)=p$ (UV form) and (b) $\omega(p)=M^{2} / p$ (IR form), respectively; see [31] for more details.

parameter of confinement in the gauge (69). This analysis also shows that the most efficient way to obtain the Polyakov loop is to carry out a so-called background field calculation with a temporal background field $a_{0}(\mathbf{x})=\left\langle A_{0}(\mathbf{x})\right\rangle$ chosen in the Polyakov gauge and then calculate the effective potential $e\left[a_{0}\right]$ of that background field. From the minimum $\bar{a}_{0}$ of this potential one evaluates the Polyakov loop $P\left[\left\langle A_{0}\right\rangle\right]=P\left[\bar{a}_{0}\right]$, which can then serve as the order parameter of confinement.

Such a calculation was done a long time ago in [61], where the effective potential $e\left[a_{0}\right]$ was calculated in one-loop perturbation theory. The result is shown in Figure 15(a). The potential is periodic due to center symmetry. The minimum of the potential occurs at the vanishing background field, which gives $P\left[a_{0}=0\right]=1$ corresponding to the deconfined phase.
This is, of course, expected due to the use of perturbation theory. Below, I present the results of a nonperturbative evaluation of $e\left[a_{0}\right]$ in the Hamiltonian approach in Coulomb gauge.

At first sight it seems that the Polyakov loop cannot be calculated in the Hamiltonian approach due to the use of the Weyl gauge $A_{0}=0$. However, we can now use the alternative Hamiltonian approach to finite temperature introduced above, where the temperature is introduced by compactifying a spatial dimension. Here, we compactify the $x_{3}$-axis and consequently put also the background field along this axis, $\mathbf{a}=a \mathbf{e}_{3}$. In the Hamiltonian approach, the effective potential of a spatial background field a can be easily calculated by minimizing the expectation value of the Hamiltonian under the constraint $\langle\mathbf{A}\rangle=\mathbf{a}$. The resulting energy $\langle H\rangle_{\mathbf{a}}=L^{2} \ell e(\mathbf{a})$ is then (up to the spatial volume factor) the effective potential. 
So the effective potential $e(a)$ is nothing but the pseudo energy density considered earlier, but now calculated in a background gauge with the constraint $\langle\mathbf{A}\rangle=\mathbf{a}$.

6.3. The Effective Potential of the Polyakov Loop. After lengthy calculations exploiting the gluon gap equation (10), one finds for the effective potential of the Polyakov loop the following expression:

$$
e(a, L)=\sum_{\sigma} \frac{1}{L} \sum_{n=-\infty}^{\infty} \int \frac{d^{2} p_{\perp}}{(2 \pi)^{2}}\left(\omega\left(\mathbf{p}^{\sigma}\right)-\chi\left(\mathbf{p}^{\sigma}\right)\right),
$$

where $\omega(p)$ is the gluon energy and $\chi(p)$ is the ghost loop. These quantities have to be taken with the momentum variable

$$
\mathbf{p}^{\sigma}=\mathbf{p}_{\perp}+\left(p_{n}-\sigma \cdot a\right) \mathbf{e}_{3}
$$

where $\mathbf{p}_{\perp}$ is the momentum corresponding to the two uncompactified space dimensions while $p_{n}=2 \pi n / L$ is the Matsubara frequency resulting from the compactification of the third dimension. Furthermore, $\sigma \cdot a \equiv \sigma^{b} a^{b}$ denotes the product of the color background field with the root vectors $\sigma^{b}$ of the gauge group. Equation (71) includes also the summation over the roots $\sigma$ of the gauge group. In [31, 62], the effective potential (71) was explicitly calculated using for $\omega(p)$ and $\chi(p)$, the results from the variational calculation in Coulomb gauge at zero-temperature [25]. This represents certainly an approximation since, in principle, one should use the finite-temperature solutions obtained in [55].

Before I present the full results let me ignore the ghost loop $\chi(p)$ in (71) and consider the ultraviolet and infrared limit of the gluon energy. If we choose the ultraviolet limit $\omega(p)=p$, we obtain from $(71)$ with $\chi(p)=0$ precisely the Weiss potential, shown in Figure 15(a), which corresponds to the deconfined phase. Choosing for the gluon energy its infrared limit $\omega(p)=M^{2} / p$, one finds from (71) with $\chi(p)=$ 0 the (center symmetric) potential shown in Figure 15(b). From its center symmetric minimum $\bar{a}=\pi / L$ one finds a vanishing Polyakov loop $P[\bar{a}]=0$ corresponding to the confined phase. Obviously, the deconfining phase transition results from the interplay between the confining infrared and the deconfining ultraviolet potentials. Choosing for the gluon energy the sum of the UV- and IR-parts $\omega(p)=p+M^{2} / p$, which can be considered as an approximation to the Gribov formula (20), one has to add the UV and IR potentials and finds a phase transition at a critical temperature $T_{c}=\sqrt{3} M / \pi$. With the Gribov mass $M \approx 880 \mathrm{MeV}$ this gives a critical value of $T_{c} \approx 485 \mathrm{MeV}$ for the color group SU(2), which is much too high as compared to the lattice value of $312 \mathrm{MeV}$ [63]. One can show analytically $[31,62]$ that the neglect of the ghost loop $\chi(p)=0$ shifts the critical temperature to higher values. If one uses for the gluon energy $\omega(p)$ the Gribov formula (20) and includes the ghost loop $\chi(p)$, one finds the effective potential shown in Figure 16(a), which shows a second-order phase transition and gives a transition temperature of $T_{c} \approx 269 \mathrm{MeV}$ for the gauge group $\mathrm{SU}(2)$, which is in the right ballpark. The Polyakov loop $P[\bar{a}]$ calculated from the minimum $\bar{a}$ of the effective potential $e(a, L)(71)$ is plotted in Figure 17(a) as function of the temperature. After the phase transition it approaches rather rapidly (at about $T / T_{c} \sim 1.1$ ) its limiting value $\langle P\rangle=1$ contrary to the lattice results. Such behavior is also observed in analogous calculations in the path-integral approach in Landau gauge; see, for example, [13, 14]. As shown in [14], the Polyakov loop approaches less rapidly its limiting value when two-loop contributions are included. This refers also to the Polyakov loop obtained for the gauge group SU(3) shown in Figure 16(b).

The effective potential for the gauge group $\mathrm{SU}(3)$ can be reduced to that of the $\mathrm{SU}(2)$ group by noticing that the $\mathrm{SU}(3)$ algebra consists of three $\mathrm{SU}(2)$ subalgebras characterized by the three positive roots $\sigma=(1,0),(1 / 2, \sqrt{3} / 2),(1 / 2,-\sqrt{3} / 2)$. One finds

$$
e_{\mathrm{SU}(3)}(a, L)=\sum_{\boldsymbol{\sigma}>0} e_{\mathrm{SU}(2)}[\boldsymbol{\sigma}](a, L)
$$

The resulting effective potential for SU(3) is shown in Figure 18 as function of the components of the background field in the Cartan algebra, $a_{3}$ and $a_{8}$. Above and below $T_{c}$ the absolute minima of the potential occur in both cases for $a_{8}=$ 0 because of charge conjugation invariance. Cutting the twodimensional potential surface at $a_{8}=0$, one finds the effective potential shown in Figure 16(b), which shows a first-order phase transition with a critical temperature of $T_{c} \approx 283 \mathrm{MeV}$. The first-order nature of the $\mathrm{SU}(3)$ phase transition is also seen in Figure 17(b), where the Polyakov loop $P[\bar{a}]$ is shown as function of the temperature.

6.4. The Dual Quark Condensate. The dual quark condensate was originally introduced in [64] and was discussed in a more general context in [65]. This quantity has been calculated on the lattice $[66,67]$, in the functional renormalization group approach [68] and in the Dyson-Schwinger approach [69]. The dual condensate is defined by

$$
\Sigma_{n}=\int_{0}^{2 \pi} \frac{d \varphi}{2 \pi} \exp (-\mathrm{i} n \varphi)\langle\bar{\psi} \psi\rangle_{\varphi},
$$

where $\langle\bar{\psi} \psi\rangle_{\varphi}$ is the quark condensate calculated with the $U(1)$-valued boundary condition:

$$
\psi\left(x^{0}+\frac{L}{2}, \mathbf{x}\right)=\mathrm{e}^{\mathrm{i} \varphi} \psi\left(x^{0}-\frac{L}{2}, \mathbf{x}\right) .
$$

For $\varphi=\pi$ these boundary conditions reduce to the usual finite-temperature boundary conditions of the quark field in the functional integral representation of the partition function; see (44a) and (44b). On the lattice it is not difficult to show that the quantity $\Sigma_{n}$ (74) represents the vacuum expectation value of the sum of all closed Wilson loops winding precisely $n$-times around the compactified time axis. In particular, the quantity $\Sigma_{1}$ represents the expectation value of all closed loops winding precisely once around the compactified time axis and is therefore called the dressed Polyakov loop. The phase in the boundary condition (75) can be absorbed into an imaginary chemical potential:

$$
\mu=\mathrm{i} \frac{\pi-\varphi}{L}
$$




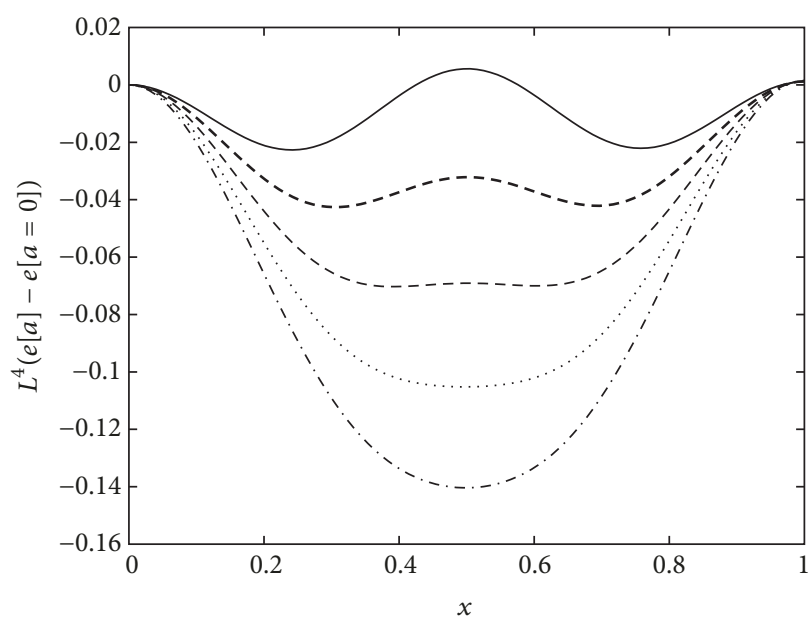

(a)

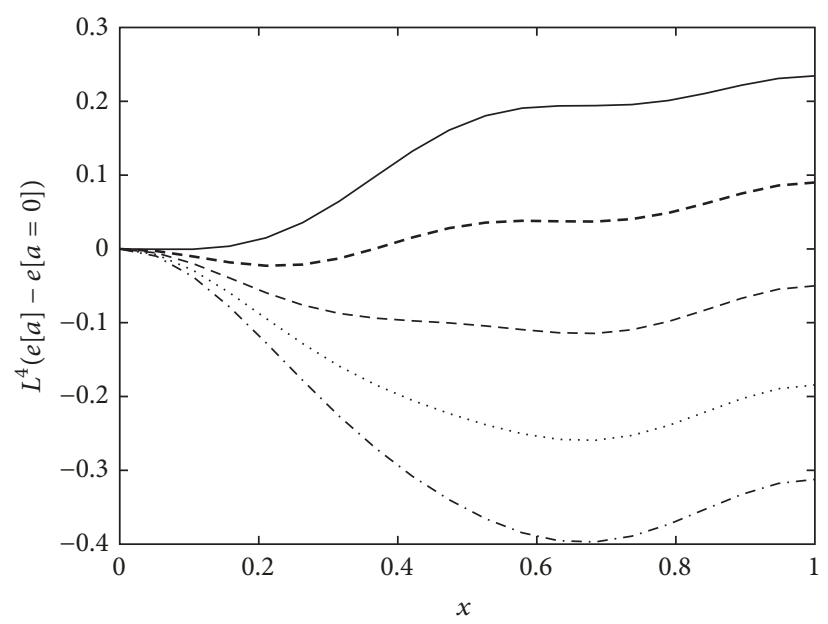

(b)

FIGURE 16: Effective potential of the Polyakov loop (71) as function of the background field $x=a_{3} L / 2 \pi$ at various temperatures, for the gauge groups (a) $\mathrm{SU}(2)$ and (b) $\mathrm{SU}(3)$.

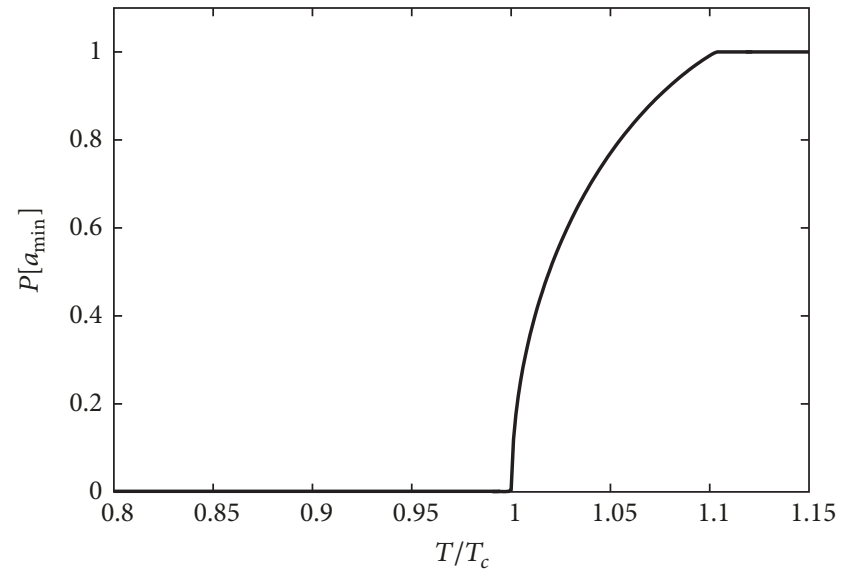

(a)

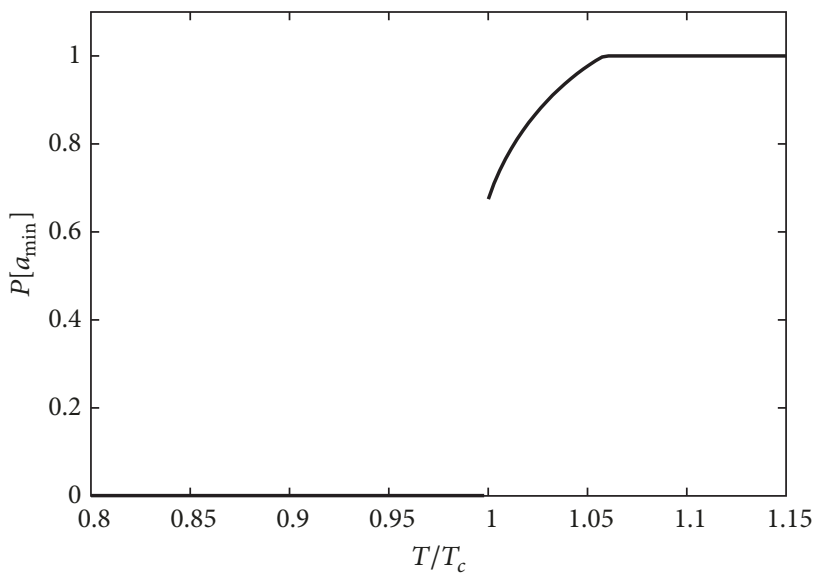

(b)

FIgUre 17: The Polyakov loop as function of the temperature (a) for SU(2) and (b) for SU(3).

for fermion fields satisfying the usual antisymmetric boundary condition $\psi\left(x^{0}+L / 2, \mathbf{x}\right)=-\psi\left(x^{0}-L / 2, \mathbf{x}\right)$. In the Hamiltonian approach to finite temperatures of [19], where the compactified time axis has become the third spatial axis, the phase-dependent boundary condition (75) or equivalently the imaginary chemical potential (76) manifests itself in the momentum variable along the (compactified) three axes, which reads

$$
p_{3}=p_{n}+\mathrm{i} \mu=\frac{2 \pi n+\varphi}{L}, \quad p_{n}=\frac{2 n+1}{L} \pi,
$$

where $p_{n}$ is the usual fermionic Matsubara frequency [see (61)]. Using the zero-temperature quark mass function $M(p)$ calculated in [30], one finds in the Hamiltonian approach to QCD of [50] for the dual quark condensate after Poisson resummation the leading expression [56]:

$$
\Sigma_{n}=-\frac{N}{\pi^{2}} \int_{0}^{\infty} d p \frac{p^{2} M(p)}{\sqrt{p^{2}+M^{2}(p)}}\left[\delta_{n 0}+\frac{\sin (n \beta p)}{n \beta p}\right],
$$

where $N$ denotes the number of colors. In the same way, one can compute the quark condensate $\langle\bar{\psi} \psi\rangle_{\varphi}$ shown in Figure 19(a). For the dressed Polyakov loop one finds the temperature behavior shown in Figure 19(b), where we also compare with the result obtained when the coupling to the transverse gauge field degrees of freedom is neglected $(g=0)$. As one observes there is no difference at small temperatures in accord with the fact that the mass function $M(p)$ has the same infrared behavior, whether the coupling to the transverse 


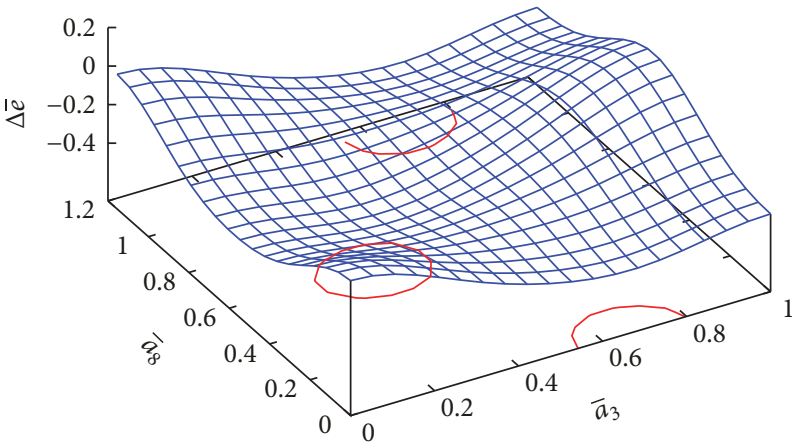

(a)

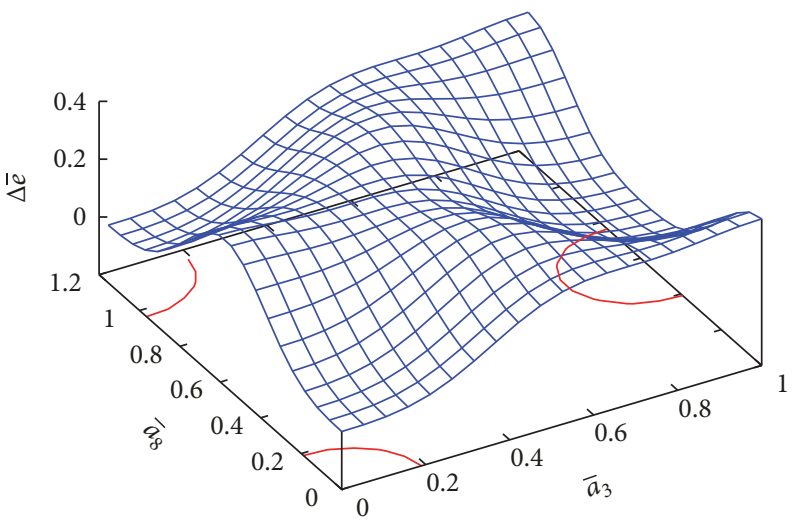

(b)

FIGURE 18: The effective potential of the Polyakov loop for the gauge group SU(3) as function of the two Cartan components of the background field $x=a_{3} L / 2 \pi$ and $y=a_{8} L / 2 \pi$ for (a) $T<T_{c}$ and (b) $T>T_{c}$.

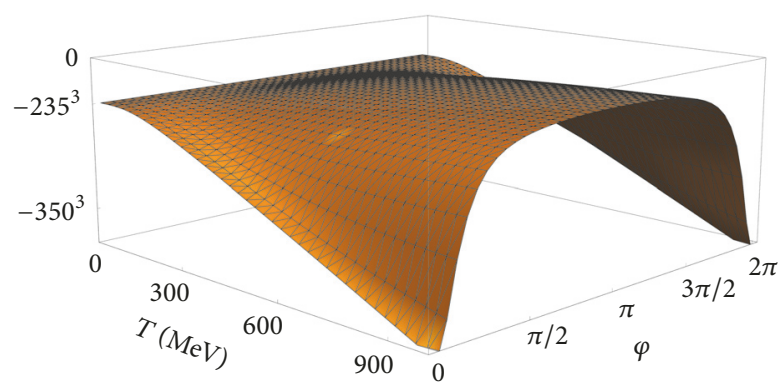

(a)

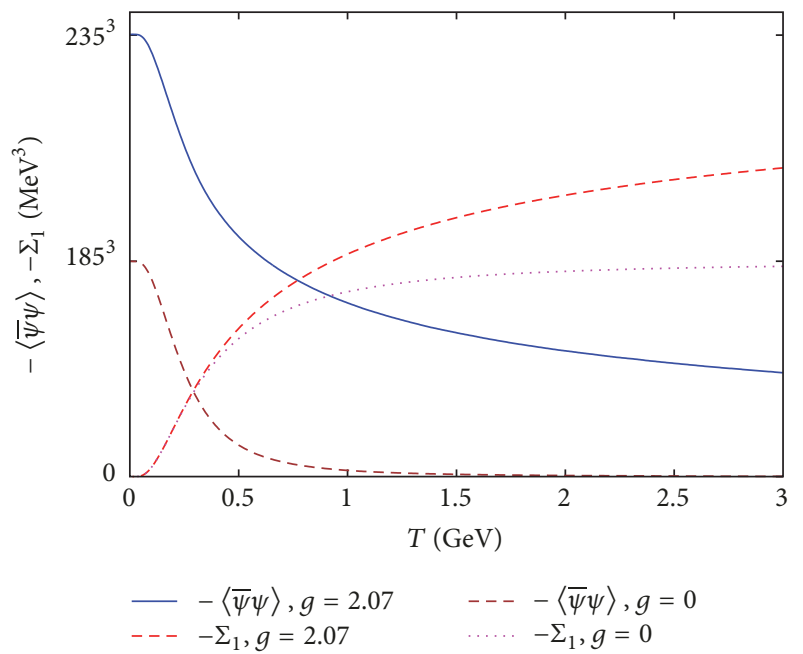

(b)

Figure 19: (a) Chiral quark condensate $\langle\bar{\psi} \psi\rangle_{\varphi}$ as function of the temperature $T$ and the phase $\varphi$ of the boundary condition (75). (b) Chiral and dual quark condensate as function of the temperature. Results are presented for both a coupling of $g \simeq 2.1$ and $g=0$.

gluons is included or not. The slower UV decrease of the full mass function causes the dual condensate to reach its hightemperature limit:

$$
\begin{aligned}
\lim _{L \rightarrow 0} \Sigma_{1} & =-\frac{N}{\pi^{2}} \int_{0}^{\infty} d p \frac{p^{2} M(p)}{\sqrt{p^{2}+M^{2}(p)}} \\
& =\lim _{L \rightarrow \infty}\langle\bar{\psi} \psi\rangle_{\varphi=\pi}
\end{aligned}
$$

only very slowly. We expect, however, that this limit is reached faster when the finite-temperature solutions are used. This will presumably also convert the crossover obtained for the chiral condensate, see Figure 19(b), into a true phase transition as expected for chiral quarks. From the inflexion points of the chiral and dual condensate one extracts the values of $T_{\chi}^{\mathrm{pc}} \simeq 170 \mathrm{MeV}$ and $T_{c}^{\mathrm{pc}} \simeq 198 \mathrm{MeV}$ for the pseudocritical temperatures of the chiral and deconfinement transition, respectively. For comparison, one finds on the lattice for realistic quark masses $T_{\chi}^{\mathrm{pc}} \simeq 155 \mathrm{MeV}$ and $T_{c}^{\mathrm{pc}} \simeq$ $165 \mathrm{MeV}[70,71]$.

\section{Conclusions}

In my talk, I have presented some recent results obtained within the Hamiltonian approach to QCD in Coulomb gauge. I have first shown that the so-called Coulomb string tension is not related to the temporal but to the spatial string tension. This relation explains the finite-temperature behavior of the Coulomb string tension, namely, the fact that it does not disappear but even increases above the deconfinement transition. I have then studied the quark sector of QCD in Coulomb gauge using a Slater determinant ansatz for the quark wave functional, which includes in particular the quark-gluon coupling by two different Dirac structures. Our calculations show that there is no spontaneous breaking of chiral symmetry 
when the (linearly rising) infrared part of the Coulomb potential is excluded. Furthermore, choosing the Coulomb string tension from the lattice data we can reproduce the phenomenological value of the quark condensate when the coupling of the quarks to the transverse gluon is included.

I have then extended the Hamiltonian approach to QCD in Coulomb gauge to finite temperatures by compactifying a spatial dimension. Within this approach, I have calculated the effective potential of the Polyakov loop as well as the chiral and dual quark condensate as function of the temperature. Using our zero-temperature solution as input, from the Polyakov loop we predict a critical temperature for the deconfinement phase transition of about $T_{c} \sim 275 \mathrm{MeV}$ for $\mathrm{SU}(2)$, and $T_{c} \sim 280 \mathrm{MeV}$ for SU(3). Furthermore, the correct order of the phase transition was found for SU(2) and $\mathrm{SU}(3)$. For full QCD our calculations of the dual and chiral quark condensate predict pseudocritical temperatures of $T_{\chi}^{\mathrm{pc}} \simeq 170 \mathrm{MeV}$ for the chiral and $T_{c}^{\mathrm{pc}} \simeq 198 \mathrm{MeV}$ for the deconfinement transition. In all these finite-temperature calculations the zero-temperature variational solutions were used as input, which is likely the reason that the critical temperatures currently obtained are too high as compared to lattice data. The solution of the variational principle at finite temperature will be the next step in our investigation of the QCD phase diagram.

\section{Disclosure}

This talk was given by H. Reinhardt at "5th Winter Workshop on Non-Perturbative Quantum Field Theory," 22-24 March 2017, Sophia-Antipolis, France.

\section{Conflicts of Interest}

The authors declare that there are no conflicts of interest regarding the publication of this paper.

\section{Acknowledgments}

This work was supported in part by DFG-RE856/9-2 and by DFG-RE856/10-1.

\section{References}

[1] C. Gattringer and K. Langfeld, "Approaches to the sign problem in lattice field theory," International Journal of Modern Physics A, vol. 31, no. 22, Article ID 1643007, 2016.

[2] C. S. Fischer, "Infrared properties of QCD from DysonSchwinger equations," Journal of Physics G: Nuclear and Particle Physics, vol. 32, no. 8, article no. R02, pp. R253-R291, 2006.

[3] R. Alkofer and L. von Smekal, "The infrared behaviour of QCD Green's functions: confinement, dynamical symmetry breaking, and hadrons as relativistic bound states," Physics Reports, vol. 353, no. 5-6, pp. 281-465, 2001.

[4] D. Binosi and J. Papavassiliou, "Pinch technique: Theory and applications," Physics Reports, vol. 479, no. 1-6, pp. 1-152, 2009.

[5] P. Watson and H. Reinhardt, "Propagator Dyson-Schwinger equations of Coulomb gauge Yang-Mills theory within the first order formalism," Physical Review D: Particles, Fields,
Gravitation and Cosmology, vol. 75, no. 4, Article ID 045021, 2007.

[6] P. Watson and H. Reinhardt, "Two-point functions of Coulomb gauge Yang-Mills theory," Physical Review D: Particles, Fields, Gravitation and Cosmology, vol. 77, no. 2, Article ID 025030, 2008.

[7] P. Watson and H. Reinhardt, "Slavnov-Taylor identities in Coulomb gauge Yang-Mills theory," The European Physical Journal $C$, vol. 65, no. 3, pp. 567-585, 2010.

[8] J. M. Pawlowski, "Aspects of the functional renormalisation group," Annals of Physics, vol. 322, no. 12, pp. 2831-2915, 2007.

[9] H. Gies, Introduction to the Functional RG and Applications to Gauge Theories, vol. 852 of Lecture Notes in Physics, Springer, Heidelberg, Berlin, Germany, 2012.

[10] C. Feuchter and H. Reinhardt, "Variational solution of the YangMills Schrödinger equation in Coulomb gauge," Physical Review D: Particles, Fields, Gravitation and Cosmology, vol. 70, Article ID 105021, 2004.

[11] H. Reinhardt and C. Feuchter, "Yang-Mills wave functional in Coulomb gauge," Physical Review D: Particles, Fields, Gravitation and Cosmology, vol. 71, no. 10, 105002, 6 pages, 2005.

[12] M. Quandt, H. Reinhardt, and J. Heffner, "Covariant variational approach to Yang-Mills theory," Physical Review D: Particles, Fields, Gravitation and Cosmology, vol. 89, no. 6, Article ID 065037, 2014.

[13] M. Quandt and H. Reinhardt, "Covariant variational approach to Yang-Mills theory at finite temperatures," Physical Review D: Particles, Fields, Gravitation and Cosmology, vol. 92, no. 2, 025051, 18 pages, 2015.

[14] U. Reinosa, J. Serreau, M. Tissier, and N. Wschebor, "Deconfinement transition in SU(2) Yang-Mills theory: A two-loop study," Physical Review D: Particles, Fields, Gravitation and Cosmology, vol. 91, no. 4, Article ID 045035, 2015.

[15] V. N. Gribov, "Quantization of non-Abelian gauge theories," Nuclear Physics B, vol. 139, no. 1-2, pp. 1-19, 1978.

[16] F. E. Canfora, D. Dudal, I. F. Justo, P. Pais, L. Rosa, and D. Vercauteren, "Effect of the Gribov horizon on the Polyakov loop and vice versa," The European Physical Journal C, vol. 75, no. 7, article no. 326, 2015.

[17] H. Reinhardt, D. Campagnari, J. Heffner, M. Quandt, and P. Vastag, "Hamiltonian approach to QCD in Coulomb gauge: From the vacuum to finite temperatures," in Proceedings of the 4th International Conference on New Frontiers in Physics, ICNFP 2015, Greece, August 2015.

[18] H. Reinhardt, G. Burgio, D. Campagnari et al., "Hamiltonian approach to QCD in Coulomb gauge at zero and finite temperature," in Proceedings of the 12Ith Quark Confinement and the Hadron Spectrum, Greece, September 2017.

[19] H. Reinhardt, "Hamiltonian finite-temperature quantum field theory from its vacuum on partially compactified space," Physical Review D: Particles, Fields, Gravitation and Cosmology, vol. 94, no. 4, Article ID 045016, 2016.

[20] N. H. Christ and T. D. Lee, "Operator ordering and Feynman rules in gauge theories," Physical Review D: Particles, Fields, Gravitation and Cosmology, vol. 22, no. 4, pp. 939-958, 1980.

[21] D. Schütte, "Nonperturbative many-body techniques applied to a Yang-Mills field theory," Physical Review D: Particles, Fields, Gravitation and Cosmology, vol. 31, no. 4, pp. 810-821, 1985.

[22] A. P. Szczepaniak and E. S. Swanson, "Coulomb gauge QCD, confinement, and the constituent representation," Physical Review D: Particles, Fields, Gravitation and Cosmology, vol. 65, no. 2, Article ID 025012, 2002. 
[23] J. Greensite, H. Matevosyan, S. Olejník, M. Quandt, H. Reinhardt, and A. P. Szczepaniak, "Testing proposals for the YangMills vacuum wavefunctional by measurement of the vacuum," Physical Review D: Particles, Fields, Gravitation and Cosmology, vol. 83, no. 11, Article ID 114509, 2011.

[24] J. Heffner, H. Reinhardt, and D. R. Campagnari, "Deconfinement phase transition in the Hamiltonian approach to YangMills theory in Coulomb gauge," Physical Review D: Particles, Fields, Gravitation and Cosmology, vol. 85, no. 12, Article ID 125029, 2012.

[25] D. Epple, H. Reinhardt, and W. Schleifenbaum, "Confining solution of the Dyson-Schwinger equations in Coulomb gauge," Physical Review D: Particles, Fields, Gravitation and Cosmology, vol. 75, no. 4, Article ID 045011, 2007.

[26] M. Leder, J. M. Pawlowski, H. Reinhardt, and A. Weber, "Hamiltonian flow in Coulomb gauge Yang-Mills theory," Physical Review D: Particles, Fields, Gravitation and Cosmology, vol. 83, no. 2, Article ID 025010, 2011.

[27] C. Feuchter and H. Reinhardt, "The Yang-Mills vacuum in Coulomb gauge in $D=2+1$ dimensions," Physical Review D: Particles, Fields, Gravitation and Cosmology, vol. 77, no. 8, Article ID 085023, 2008.

[28] L. Moyaerts, A Numerical Study of Quantum Forces: Casimir Effect, Vortices and Coulomb Gauge Yang-Mills Theory [PhD thesis], 2004.

[29] G. Burgio, M. Quandt, H. Reinhardt, and H. Vogt, "Coulomb versus physical string tension on the lattice," Physical Review D: Particles, Fields, Gravitation and Cosmology, vol. 92, no. 3, Article ID 034518, 2015.

[30] D. R. Campagnari, E. Ebadati, H. Reinhardt, and P. Vastag, "Revised variational approach to QCD in Coulomb gauge," Physical Review D: Particles, Fields, Gravitation and Cosmology, vol. 94, no. 7, Article ID 074027, 2016.

[31] H. Reinhardt and J. Heffner, "Effective potential of the confinement order parameter in the Hamiltonian approach," Physical Review D: Particles, Fields, Gravitation and Cosmology, vol. 88, no. 4, Article ID 045024, 2013.

[32] H. Reinhardt, "Dielectric function of the QCD vacuum," Physical Review Letters, vol. 101, no. 6, 2008.

[33] S. Mandelstam, "Vortices and quark confinement in nonAbelian gauge theories," Physics Reports, vol. 23, no. 3, pp. 245249, 1976 .

[34] G. 't Hooft, “The topological mechanism for permanent quark confinement in a non-abelian gauge theory," Physica Scripta, vol. 25, no. 1, pp. 133-142, 1982.

[35] G. Burgio, M. Quandt, and H. Reinhardt, "Coulomb-gauge gluon propagator and the Gribov formula," Physical Review Letters, vol. 102, no. 3, Article ID 032002, 2009.

[36] D. R. Campagnari and H. Reinhardt, "Non-Gaussian wave functionals in Coulomb gauge Yang-Mills theory," Physical Review D: Particles, Fields, Gravitation and Cosmology, vol. 82, no. 10, Article ID 105021, 2010.

[37] P. de Forcrand and J. E. Hetrick, "The continuum limit of the lattice Gribov problem, and a solution based on Hodge decomposition," Nuclear Physics B (Proceedings Supplements), vol. 42, no. 1-3, pp. 861-866, 1995.

[38] A. Sternbeck and M. Müller-Preussker, "Lattice evidence for the family of decoupling solutions of Landau gauge Yang-Mills theory," Physics Letters B, vol. 726, no. 1-3, pp. 396-403, 2013.

[39] P. Cooper and D. Zwanziger, "Spectral decomposition of the ghost propagator and a necessary condition for confinement,"
Physical Review D: Particles, Fields, Gravitation and Cosmology, vol. 93, no. 10, Article ID 105024, 2016.

[40] G. Burgio, M. Quandt, H. Reinhardt, and H. Vogt, "Gribov horizon and Gribov copies effect in lattice Coulomb gauge," Physical Review D: Particles, Fields, Gravitation and Cosmology, vol. 95, no. 1, Article ID 014503, 2017.

[41] D. Zwanziger, "No confinement without Coulomb confinement," Physical Review Letters, vol. 90, 2003.

[42] J. Greensite, Š. Olejník, and D. Zwanziger, "Coulomb energy, remnant symmetry, and the phases of non-Abelian gauge theories," Physical Review D: Particles, Fields, Gravitation and Cosmology, vol. 69, no. 7, Article ID 074506, 2004.

[43] A. Voigt, E.-M. Ilgenfritz, M. Müller-Preussker, and A. Sternbeck, "Effective Coulomb potential in SU(3) lattice yang-mills theory," Physical Review D: Particles, Fields, Gravitation and Cosmology, vol. 78, no. 1, Article ID 014501, 2008.

[44] J. Greensite, An Introduction to the Confinement Problem, vol. 821 of Lecture Notes in Physics, Springer-Verlag, Berlin, Germany, 2011.

[45] H. Reinhardt, "Topology of center vortices," Nuclear Physics B, vol. 628, no. 1-2, pp. 133-166, 2002.

[46] P. De Forcrand and M. D'Elia, "Relevance of center vortices to QCD," Physical Review Letters, vol. 82, no. 23, pp. 4582-4585, 1999.

[47] L. Del Debbio, M. Faber, J. Greensite, and S. Olejnk, "Detection of center vortices in the lattice Yang-Mills vacuum," Physical Review D: Particles, Fields, Gravitation and Cosmology, vol. 58, Article ID 094501, 1998.

[48] K. Langfeld, H. Reinhardt, and O. Tennert, "Confinement and scaling of the vortex vacuum of SU(2) lattice gauge theory," Physics Letters. B. Particle Physics, Nuclear Physics and Cosmology, vol. 419, no. 1-4, pp. 317-321, 1998.

[49] M. Pak and H. Reinhardt, "Quark sector of the QCD groundstate in Coulomb gauge," Physical Review D: Particles, Fields, Gravitation and Cosmology, vol. 88, no. 12, Article ID 125021, 2013.

[50] P. Vastag, H. Reinhardt, and D. Campagnari, "Improved variational approach to QCD in Coulomb gauge," Physical Review D: Particles, Fields, Gravitation and Cosmology, vol. 93, no. 6, Article ID 065003, 2016.

[51] J. R. Finger and J. E. Mandula, "Quark pair condensation and chiral symmetry breaking in QCD," Nuclear Physics B, vol. 199, no. 1, pp. 168-188, 1982.

[52] S. L. Adler and A. C. Davis, "Chiral symmetry breaking in Coulomb gauge QCD," Nuclear Physics B, vol. 244, no. 2, pp. 469-491, 1984.

[53] R. Alkofer and P. A. Amundsen, "Chiral symmetry breaking in an instantaneous approximation to Coulomb gauge QCD," Nuclear Physics B, vol. 306, no. 2, pp. 305-342, 1988.

[54] H. Reinhardt, D. R. Campagnari, and A. P. Szczepaniak, "Variational approach to Yang-Mills theory at finite temperatures," Physical Review D: Particles, Fields, Gravitation and Cosmology, vol. 84, no. 4, Article ID 045006, 2011.

[55] J. Heffner and H. Reinhardt, "Finite-temperature Yang-Mills theory in the Hamiltonian approach in Coulomb gauge from a compactified spatial dimension," Physical Review D: Particles, Fields, Gravitation and Cosmology, vol. 91, no. 8, Article ID 085022, 2015.

[56] H. Reinhardt and P. Vastag, "Chiral and deconfinement phase transition in the Hamiltonian approach to QCD in Coulomb gauge," Physical Review D: Particles, Fields, Gravitation and Cosmology, vol. 94, no. 10, 2016. 
[57] B. Svetitsky, "Symmetry aspects of finite-temperature confinement transitions," Physics Reports, vol. 132, no. 1, pp. 1-53, 1986.

[58] J. Braun, H. Gies, and J. M. Pawlowski, "Quark confinement from colour confinement," Physics Letters B, vol. 684, no. 4-5, pp. 262-267, 2010.

[59] M. Florian and J. M. Pawlowski, Confinement in Polyakov Gauge, 2008.

[60] M. Quandt and H. Reinhardt, "Covariant variational approach to Yang-Mills theory: effective potential of the Polyakov loop," Physical Review D: Particles, Fields, Gravitation and Cosmology, vol. 94, no. 6, 065015, 17 pages, 2016.

[61] N. Weiss, "Effective potential for the order parameter of gauge theories at finite temperature," Physical Review D: Particles, Fields, Gravitation and Cosmology, vol. 24, no. 2, pp. 475-480, 1981.

[62] H. Reinhardt and J. Heffner, "The effective potential of the confinement order parameter in the Hamiltonian approach," Physics Letters B, vol. 718, no. 2, pp. 672-677, 2012.

[63] B. Lucini, M. Teper, and U. Wenger, "The high temperature phase transition in $\mathrm{SU}(\mathrm{N})$ gauge theories," Journal of High Energy Physics, vol. 2004, no. 01, pp. 061-061, 2004.

[64] C. Gattringer, "Linking confinement to spectral properties of the dirac operator," Physical Review Letters, vol. 97, no. 3, Article ID 032003, 2006.

[65] F. Synatschke, A. Wipf, and C. Wozar, "Spectral sums of the Dirac-Wilson operator and their relation to the Polyakov loop," Physical Review D: Particles, Fields, Gravitation and Cosmology, vol. 75, no. 11, Article ID 114003, 2007.

[66] E. Bilgici, F. Bruckmann, C. Gattringer, and C. Hagen, "Dual quark condensate and dressed Polyakov loops," Physical Review D: Particles, Fields, Gravitation and Cosmology, vol. 77, no. 9, Article ID 094007, 2008.

[67] B. Zhang, F. Bruckmann, Z. Fodor, C. Gattringer, and K. K. Szabó, "Dual condensate and QCD phase transition," in Proceedings of the 9th International Conference on Quark Confinement and the Hadron Spectrum, QCHS IX, pp. 170-172, Spain, September 2011.

[68] J. Braun, L. M. Haas, F. Marhauser, and J. M. Pawlowski, "Phase structure of two-flavor QCD at finite chemical potential," Physical Review Letters, vol. 106, no. 2, Article ID 022002, 2011.

[69] C. S. Fischer, A. Maas, and J. A. Müller, "Chiral and deconfinement transition from correlation functions: SU(2) vs. SU(3)," The European Physical Journal C, vol. 68, no. 1, pp. 165-181, 2010.

[70] S. Borsányi, Z. Fodor, and C. Hoelbling, "Is there still any $T_{c}$ mystery in lattice QCD? Results with physical masses in the continuum limit III," Journal of High Energy Physics, vol. 2010, article 73, 2010.

[71] A. Bazavov et al., "The chiral and deconfinement aspects of the QCD transition," Physical Review D: Particles, Fields, Gravitation and Cosmology, vol. 85, Article ID 054503, 2012. 

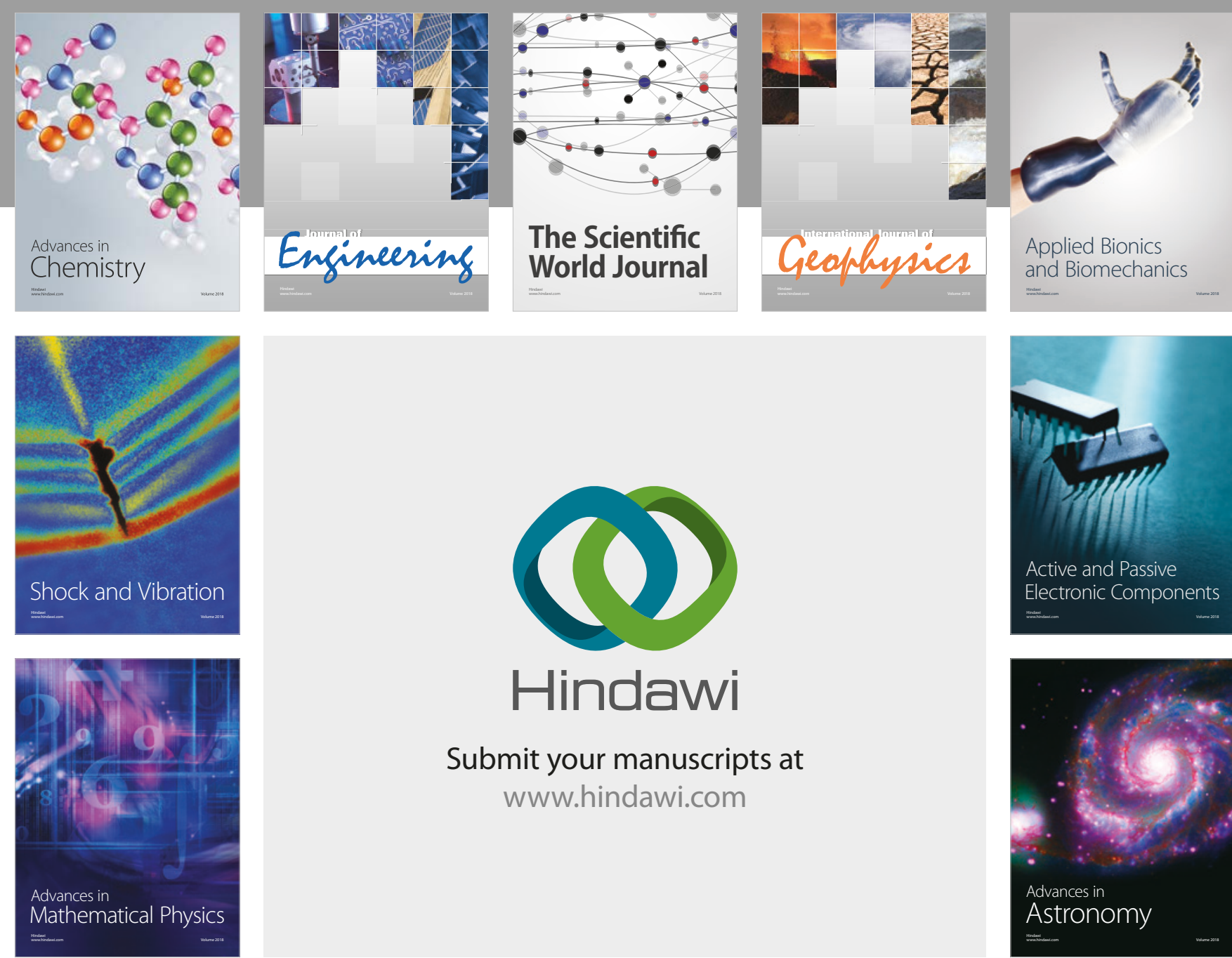

Submit your manuscripts at

www.hindawi.com

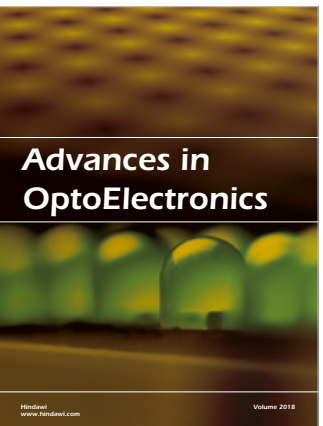

\section{Rotcting Machinery}
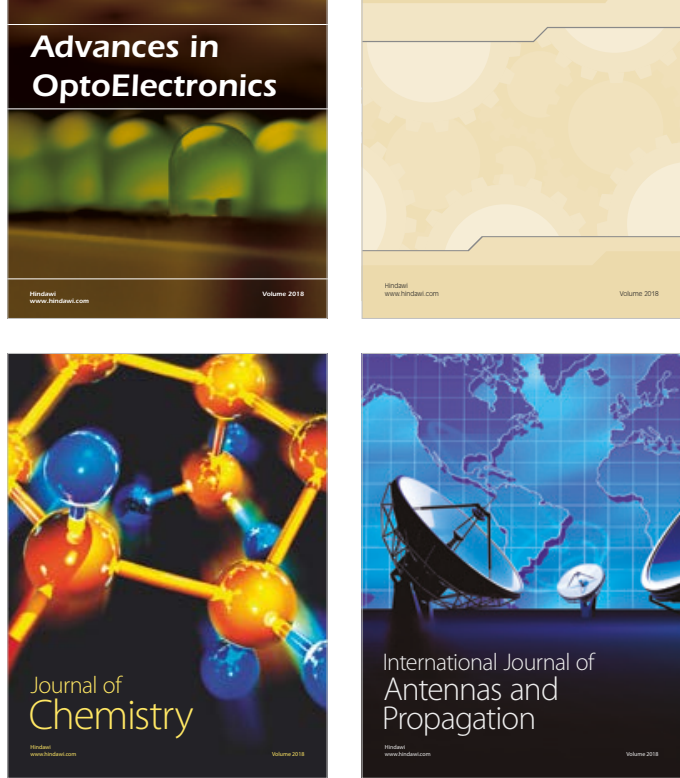

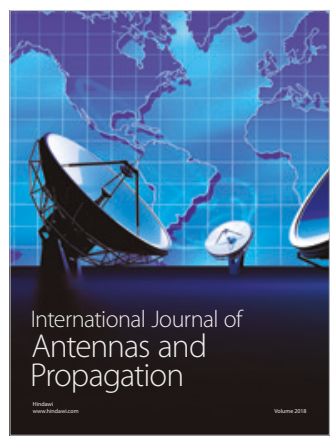

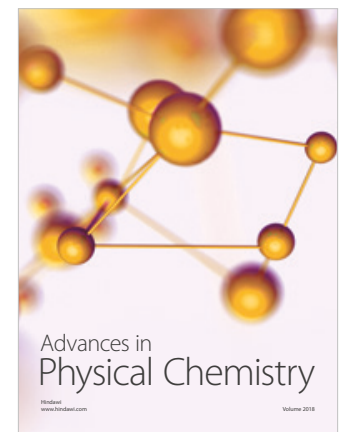

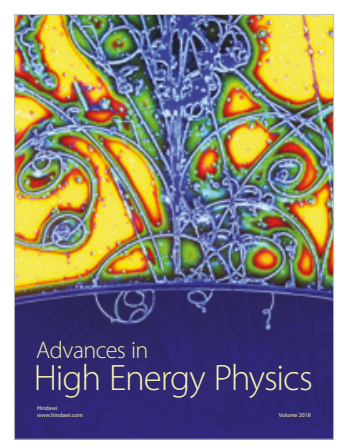

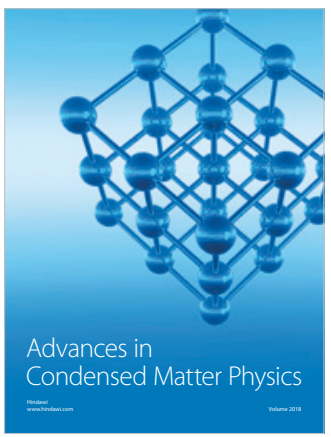

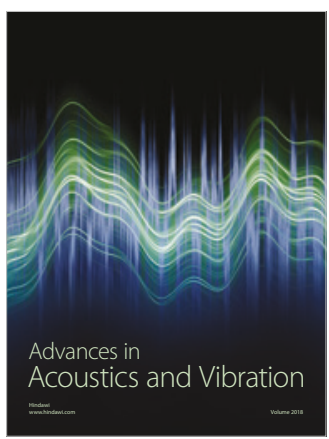

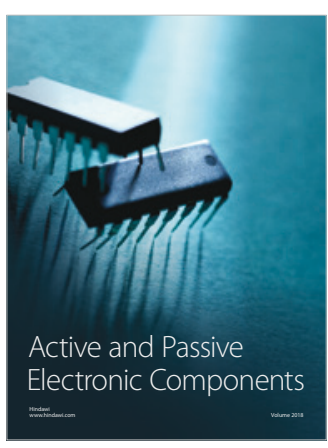
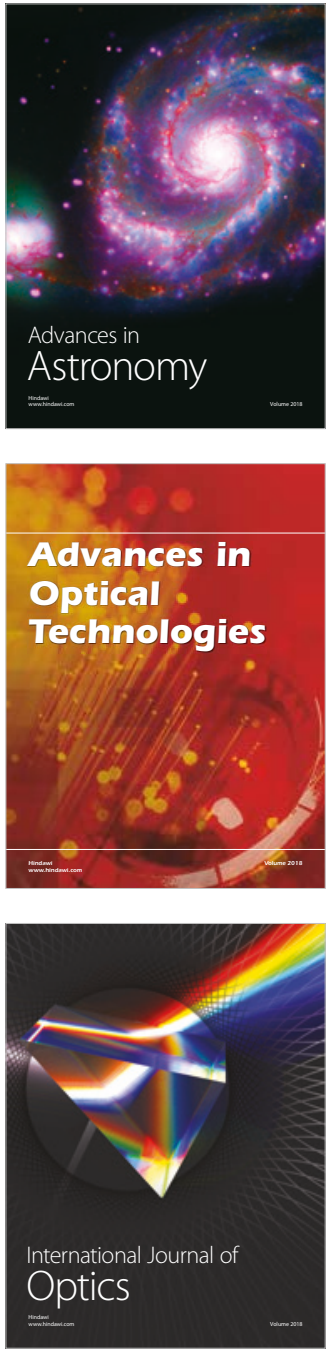